\title{
Activations in gray and white matter are modulated by uni- manual responses during within and inter-hemispheric transfer: Effects of response hand and right-handedness
}

\author{
Vaibhav A. Diwadkar ${ }^{1}{ }^{\star}$, Marcella Bellani $^{2}$, Asadur Chowdury $^{1}$, Silvia Savazzi $^{3}$, Cinzia \\ Perlini $^{2}$, Veronica Marinelli ${ }^{2}$, Giada Zoccatelli ${ }^{4}$, Franco Alessandrini ${ }^{4}$, Elisa Ciceri ${ }^{4}$, \\ Gianluca Rambaldelli ${ }^{2}$, Mirella Ruggieri ${ }^{2}$, A. Carlo Altamura ${ }^{5}$, Carlo A. Marzi ${ }^{3}$, and Paolo \\ Bambilla ${ }^{5,}$ \\ ${ }^{1}$ Dept. of Psychiatry \& Behavioral Neurosciences, Wayne State University, Tolan Park Medical \\ Building, Suite 5B, 3901 Chrysler Service Drive, Detroit MI 48301 USA \\ 2Department of Public Health and Community Medicine, Section of Psychiatry and Section of \\ Clinical Psychology, University of Verona, Verona Italy 37129 \\ ${ }^{3}$ Section of Physiology, Faculty of Medicine \& Surgery, University of Verona, Verona Italy 37129 \\ ${ }^{4}$ Neuroradiology Department, Azienda Ospedaliera Universitaria Integrata di Verona, Verona, Italy \\ 37126 \\ ${ }^{5}$ Dept. of Psychiatry, University of Milan, Milan Italy
}

\begin{abstract}
Because the visual cortices are contra-laterally organized, inter-hemispheric transfer tasks have been used to behaviorally probe how information briefly presented to one hemisphere of the visual cortex is integrated with responses resulting from the ipsi- or contra-lateral motor cortex. By forcing rapid information exchange across diverse regions, these tasks robustly activate not only gray matter regions, but also white matter tracts. It is likely that the response hand itself (dominant or non-dominant) modulates gray and white matter activations during within and inter-hemispheric transfer. Yet the role of uni-manual responses and/or right hand dominance in modulating brain activations during such basic tasks is unclear. Here we investigated how uni-manual responses with either hand modulated activations during a basic visuo-motor task (the established Poffenberger paradigm) alternating between inter- and within-hemispheric transfer conditions. In a large sample of strongly right-handed adults $(\mathrm{n}=49)$, we used a factorial combination of transfer condition [Inter vs. Within] and response hand [Dominant(Right) vs. Non-Dominant (Left)] to discover fMRI-based activations in gray matter, and in narrowly defined white matter tracts. These
\end{abstract}

\footnotetext{
*Correspondence to: Vaibhav A. Diwadkar, PhD Professor, Brain Imaging Research Division, Dept. of Psychiatry \& Behavioral Neurosciences, Wayne State University School of Medicine, Suite 5B, 3901 Chrysler Drive, Detroit MI 48201, USA, Paolo Brambilla, MD PhD, Department di Neuroscienze, e Salute Mentale, Fon-dazione IRCCS Ca', Granda Ospedale Policlinico, via Francesca Sforza 35, 20122 Milano, Italy.

Conflicts of Interest The authors have no conflicts of interest to report.

Informed Consent

All procedures followed were in accordance with the ethical standards of the responsible committee on human experimentation (institutional and national) and with the Helsinki Declaration of 1975, and the applicable revisions at the time of the investigation. Informed consent was obtained from all patients for being included in the study.
} 
tracts were identified using a priori probabilistic white matter atlases. Uni-manual responses with the right hand strongly modulated activations in gray matter, and notably in white matter. Furthermore, when responding with the left hand, activations during inter-hemispheric transfer were strongly predicted by the degree of right-hand dominance, with increased right-handedness predicting decreased fMRI activation. Finally, increasing age within the middle-aged sample was associated with a decrease in activations. These results provide novel evidence of complex relationships between uni-manual responses in right-handed subjects, and activations during within- and inter-hemispheric transfer. The results suggest that the organization of the motor system exerts sophisticated functional effects. Moreover, our evidence of activation in white matter tracts is consistent with prior studies, confirming fMRI-detectable white matter activations which are systematically modulated by experimental condition.

\section{Keywords}

Right-Handedness; Inter-hemispheric transfer; Motor organization; Poffenberger paradigm; White matter; fMRI

\section{Introduction}

Hemispheric organization is a strong organizing principle of both the motor system (Jeannerod 1988; Gazzaniga 1989; Kim et al. 1993) and the primary visual system (Tootell et al. 1998; Hellige 1996), and disordered hemispheric transfer is a feature of several neuropsychological and neurological conditions. The contra-lateral pattern of hemispheric organization (e.g., Right Hand $\rightarrow$ Left Motor Cortex; Left Visual Field $\rightarrow$ Right Visual Cortex) is strongly established in right-handed adults (Serrien et al. 2006; Toga and Thompson 2003; van den Berg et al. 2011), that is $~ 90 \%$ of the general population (Amunts et al. 1996). This lateralized organization of the sensorimotor system partially constrains the flow of information between cerebral hemispheres, most saliently in inter-hemispheric transfer tasks (Marzi 1999) where one hemisphere of the visual cortex is stimulated (e.g., Right Visual Cortex), and uni-manual responses depend on the motor cortex in the opposite hemisphere (Left Motor Cortex). In such cases, fMRI studies have suggested robust activation of both gray and white matter (Mazerolle et al. 2008). Thus controlled interhemispheric transfer tasks generate dynamic, and task-sensitive representations in the brain (Serrien et al. 2006), though these dynamics are also in play regardless of the interhemispheric demands of tasks (Gawryluk et al. 2014).

Representations of inter-hemispheric transfer (IT) may be particularly related to the lateralized organization of sensorimotor systems (Aboitiz et al. 2003). Thus:

a. When under tachistoscopic conditions, a probe is briefly presented in one visual field (leading to stimulation of the contra-lateral visual cortex), and

b. The subject must detect its presence by a uni-manual response, then

c. Patterns of brain activations may be related to the handedness of the participant, and whether the hand used in signaling the response is dominant or nondominant. 
Furthermore, IT generates different demands on the macroscopic brain network than withinhemispheric transfer (i.e., when the stimulated visual cortex and the responding motor cortex are in the same cerebral hemisphere). Thus, uni-manual responses and handedness may exert distinct effects for IT and within-hemispheric conditions.

Whether handedness mediates representations of either IT or within-hemispheric transfer is notably understudied. Here we investigated this basic question in a sample of young righthanded adults. These were the principle questions of interest: a) During IT and/or withinhemispheric transfer, do right-handed subjects activate brain regions differently when responding with their dominant hand, relative to their non-dominant hand? b) Are these activation patterns robustly and distinctly quantifiable? c) When strongly right-handed subjects respond with their non-dominant left hand, are activation patterns predicted by the degree of right-handedness and are these effects more pronounced during IT conditions?

\section{The Poffenberger Paradigm}

The behavioral costs of inter-hemispheric transfer has been extensively studied using the Poffenberger paradigm, a historically significant, visuo-motor detection task (Poffenberger 1912; Zaidel and Iacoboni 2003) with a straightforward rationale (depicted in Figure 1): A stimulus briefly presented to a single visual hemi-field (left or right) must be rapidly detected with a uni-manual (right or left hand) response. Behavioral metrics indicate subjects take between 3-6 ms longer responding when the responding motor cortex is contra-lateral to the stimulated visual cortex (Marzi et al. 1991). This delay presumably reflects conduction costs of information crossing the inter-hemispheric space (Poffenberger 1912), principally white matter pathways and inter-hemispheric commissures such as the corpus callosum (Gazzaniga 2000; Schulte et al. 2005). For instance, in patients with agenesis or surgical section of the corpus callosum, response latencies during IT are substantially longer (Savazzi et al. 2007; Mooshagian et al. 2009; Berlucchi et al. 1995). As previously noted, in vivo studies have shown robust fMRI-measured activation in both gray and white matter (Mazerolle et al. 2010; Mazerolle et al. 2008; Gawryluk et al. 2011a; Martuzzi et al. 2006).

We hypothesized asymmetric effects of uni-manual responses on IT-related activations assuming a) IT depends on integration across hemispheric lateralized sensory and motor cortices and $b$ ) because this sensorimotor organization itself mediates handedness. Here we extended the Poffenberger paradigm to investigate the effects of handedness on fMRImeasured IT activations in a large sample of young right-handed adults ( $\mathrm{n}=49,20 \leq$ Age $\leq$ 41 yrs.). During the task, participants responded with their right (dominant) or left hand to signal detection of briefly presented probes (to the ipsi or contralateral visual cortex). The sample size and the relatively constricted age-range were employed to amplify power and constrain potential age-related effects on callosal function (Fling and Seidler 2012). Eventrelated design (Amaro and Barker 2006; Josephs and Henson 1999) (as opposed to the previously used block designs to study IT) allowed unpredictability in experimental onsets (inter- or intra-hemispheric conditions), thereby increasing sensitivity for detecting condition-specific variations in neuronal responses. Discovery of white matter activations was restricted to stereotactic high probability white matter masks (Hua et al. 2008) preceded 
by use of narrower smoothing filters in the data processing stream. Whereas fMRI based activations in white matter are a subject of debate, evidence suggests that such activations are reliable under specific task conditions (Gawryluk et al. 2014).

\section{Materials and Methods}

\section{Subjects}

49 recruited healthy young adults from the Verona area provided informed consent to participate in the fMRI study. Subject demographic features and information on the degree of right-handedness are shown in Table 1. Handedness was established using the Edinburgh handedness inventory (Oldfield 1971), adopting a conservative scoring procedure;

Responses for the ten scale items were scored using a five-point Likert Scale (Very Left Handed: -2 to Very Right Handed: +2) (Byrne et al. 2004; Dragovic 2004). The cumulative score across participants ranged between -20 (strongly endorsing all ten items for the left hand: Completely Left Handed) and +20 (strongly endorsing all ten items for the right hand: Completely Right Handed; with a score of $\geq 14$ representing strong right-handedness). The group assessed here included only those deemed strongly right-handed based on these criteria (depicted in the heat map in Figure 2).

fMRI

MRI data were acquired on a 3-Tesla MR imaging unit Siemens Allegra system (Siemens, Erlangen, Germany) with a standard head coil. T2*-weighted images were acquired using a gradient-echo EPI-BOLD pulse sequence (TR: $2000 \mathrm{~ms}$; TE: $30 \mathrm{~ms}$; flip angle $75^{\circ}$; FOV: $92 \times 192$; 31 axial slices; thickness: $3 \mathrm{~mm}$; in-plane: $3 \mathrm{~mm}^{2}$; matrix: $\left.64 \times 64\right)$. High-resolution MPRAGE $\mathrm{T}_{1}$-weighted structural images were acquired in the same session (TR: $2300 \mathrm{~ms}$; TE: $3.93 \mathrm{~ms}$; flip angle $12^{\circ}$; FOV: 256×256; 160 axial slices; slice thickness: $1 \mathrm{~mm}$; matrix $256 \times 256)$.

During fMRI subjects were positioned with adjustable padded restraints employed for head stabilization. Stimuli were rear-projected using an IFIS-SA presentation system (MRI Devices), and subjects responded with the index finger of the Right or Left hand using a button response unit. Subjects maintained fixation on a centrally positioned marker, while probes were briefly presented (100 ms preempting saccadic eye movements) for detection. Probes were $\sim 1^{\circ}$ in size appearing at a retinal eccentricity of $\sim 7^{\circ}$ along the horizontal meridian of one or the other visual hemi-field.

Design efficiency for fMRI was maximized using event-related probe presentation (Dale 1999; Josephs and Henson 1999; Liu 2004), with jittering of successive intervals (3-6 s, randomly applied). Response hand was blocked across scans; subjects responded with the Right (dominant) or the Left (non-dominant) hand in each of two consecutively acquired scans (182 volumes each). Blocking response hand across scans was designed to preempt ancillary effects of excitatory and inhibitory signaling (Grefkes et al. 2008), and response competition (Nachev et al. 2007), processes that were tangential to the goals of the study. This overall experimental structure provided a factorial combination of Response Hand 
(Right vs. Left) and Condition (Inter- vs. Within-), elaborated in the description of contrast structure below. Thirty event trials were collected per condition.

\section{fMRI Processing}

fMRI and T1 data were processed in SPM12 using established methods for temporal (slice timing correction) followed by spatial preprocessing. First the structural and functional images were manually reoriented to AC-PC axis. The structural images were segmented to identify different tissue classes using SPM12's tissue probability maps, creating a nonlinear deformation normalization model. Motion correction and alignment to the template image was followed by co-registering the EPI images to the anatomical. The deformation model previously created was applied to the co-registered images to normalize them to the MNI space (Ashburner and Friston 2005). These warped images were smoothed using a relatively narrow $4 \mathrm{~mm}$ FWHM Gaussian filter to fulfill the statistical requirements of parametric statistics within the context of random fields approaches to imaging data, while maintaining relative localization of signal. These decisions allowed us to better fulfill our aims of activation explorations in gray and white matter (Friston et al. 1995).

First level GLM analysis employed regressors to represent Conditions, modeled as individual events convolved with a canonical hemodynamic reference waveform, with time and dispersion derivatives (to allow for peak, and subject- and voxel-wise variations respectively). The six motion parameters (three for translation and three for rotation) were included in first level models as "regressors of no interest" to filter out motion-related contributions to the signal. Each subject contributed four regressors to a two-factor second level analysis of variance, representing a factorial combination of response hand and condition. In the factorial design, within each of the Within- and Inter-hemispheric conditions, directional contrasts were employed to quantify relative differences in activation associated with each response hand with age modeled as covariate. This analytical approach allowed us to directly compare the effects of uni-manual responses in each of the inter- and within-hemispheric transfer conditions.

Significant clusters within each region were identified using AlphaSim (Ward, 2000), by estimating the minimum cluster extent for activated clusters to be rejected as false positive (noise-only) clusters (family-wise error corrected using a frequency of occurrence of minimum cluster thresholds of $a<.05 ; 10^{4}$ Monte Carlo simulations). This approach performs a Monte Carlo alpha probability simulation, computing the probability of a random field of noise (after accounting for the spatial correlations of voxels based on the image smoothness within each region of interest estimated directly from the data set) to produce a cluster of a given size, after the noise is thresholded at a given level. Thus, instead of using the individual voxel probability threshold alone in achieving the desired overall significance level, the method uses a combination of both probability thresholding and minimum cluster size thresholding. The underlying principle is that true regions of activation will tend to occur over contiguous voxels within a region of relative functional homogeneity, whereas noise has much less of a tendency to form clusters of activated voxels. This method has reliably identified activation clusters within narrowly defined motor regions (Asemi et al. 2015; Friedman et al. 2017; Diwadkar et al. 2017) during simple motor behavior, in other 
words indicating that under appropriately task-constrained acquisition and analyses, is well positioned to identify activation loci. Regional activation peaks in gray matter were then subsequently labeled (see Tables) using stereotactic region of interest maps (Maldjian et al. 2003; Tzourio-Mazoyer et al. 2002).

\section{Exploring white matter activations}

Activations in white matter were isolated using the Johns Hopkins University's (JHU) whole brain white matter probabilistic map (Zhang et al. 2010; Hua et al. 2008). This mask created using Diffusion Tensor imaging data from 28 control participants provides a stereotactic representation of reliably classifiable white matter tracts. For 3D tract reconstruction, the Fiber Assignment by Continuous Tractography (FACT) method was used with a fractional anisotropy threshold (0.2) in DTIstudio. Tracts are reconstructed using multiple regions of interest and leveraging existing anatomical knowledge, with two different templates (JHUDTI and MNI-ICBM152) used for spatial normalization and co-registration. These maps of eleven white matter tracts reveal high probabilities within a skeletal core, while probabilities are more dispersed and less reliable in vicinity of the cortex itself (and are thus excluded from the mask). Twenty distinct white matter tracts were identified in MNI space using this DTI approach, and for the present study, the map was binarized, to restrict identification of white matter related activations to regions more reliably classified within this tissue class. As with gray matter, cluster level correction was applied to identify significant clusters under the mask, with significance peaks labeled using the JHU template. The mask is overlaid in subsequent figures (see Figures 5, 7 and 9) showing results of white matter activations.

\section{Results}

\section{Behavioral Results}

Data from two subjects were lost on account of equipment error. Response latencies for one subject exceeded 3 s.d. from the group mean and were not analyzed. Therefore, mean response latencies for 46 participants were analyzed in a repeated measures analysis of covariance with response hand and visual field as within subject's factors and age and gender as covariates. The effect of response hand was significant, $F_{1,43}=8.29, p<.006$, $M S \mathrm{e}=2291.83$ (with a large effect size, partial $\eta^{2}=.162$ ), with subjects taking longer when responding with the non-dominant left hand, where a significant IT effect (greater than 0) was observed $\left(t_{45}=3.44, p<.001\right.$; Figure 3$)$. In additional analyses, we also assessed the effects of age on the latency difference within subjects between the inter-hemispheric and within-hemispheric condition (Tettamanti et al. 2002). Age did not exert significant effects on this behavioral cost function for either the Left Hand $\left(F_{1,45}=.38, p>.5, M S e=369.03\right)$ or the Right Hand $\left(F_{1,45}=.55, p>.45, M S e=432.92\right)$.

\section{fMRI Results}

fMRI results $(n=49)$ drawn from the overall factorial analysis of variance are reported in successive figures as follows: a) We report directional effects (Left Hand $\neq$ Right Hand) separately for each of the Within- and Inter-hemispheric conditions. The significant clusters represent relative differences in activation when responding with either the dominant right hand, or the non-dominant left hand during each of the conditions of the interest. Effects are 
reported in sequence for gray matter (Figure 4) and white matter (Figure 5). b) We next report results of regression analyses examining the effect of degree of right-handedness on Within- and Inter-hemispheric conditions when responding with the non-dominant left hand. Here again, effects are reported in sequence for gray matter (Figure 6) and white matter (Figure 7). c) In addition to having modeled age as a covariate in the overall factorial design, we also present age-related regressions investigating changes in response profiles in gray (Figure 8) and white matter (Figure 9) during left handed responses. These analyses provide some insight into how age affects cross handed representations in our sample.

(a) How do uni-manual responses affect patterns of activations in gray and white matter during within- and inter-hemispheric transfer?-During withinhemispheric transfer, uni-manual responses with either hand resulted in multiple loci of gray matter activations across the hemispheres, and these patterns were largely symmetric. Figure 4 depicts results in a mosaic of axial views. As seen in Figure 4a, responses with the right hand (warm colors) resulted in activations in left-hemisphere motor regions, but cerebellar activations in the ipsi-lateral, right hemisphere. Responses with the left hand resulted in activations in right-hemisphere motor regions, and left hemisphere cerebellar activations (Table 2). These activations for either hand were observed in both core motor regions (M1, SMA and the cerebellum), and multiple cortical, striatal and thalamic regions.

By comparison to the within-hemispheric condition, the inter-hemispheric transfer condition induced asymmetric activations for uni-manual responses with more representations observed for the dominant right hand in the SMA and the dPFC (see Table 3 and Figure 4b). We next depict uni-manual activations associated with within and inter-hemispheric transfer in white matter pathways.

Figure 5 depicts activations in deep white matter tracts induced by within-hemispheric transfer (Figure 5a) or inter-hemispheric transfer (Figure 5b). The conventions for depicting significance are maintained from Figure 4. As seen, responding with the right hand results in highly asymmetric patterns of activation in white matter, particularly during the IT condition (an effect considerably amplified relative to effects in gray matter). Table 4 depicts statistical information regarding loci peaks observed for each of the within and inter-hemispheric conditions.

These analyses imply that responding with the dominant right hand results in somewhat privileges activations during the inter-hemispheric transfer condition. Lateralization within the motor system in right-handers has been associated with transcallosal inhibition from left M1 to right M1, indicating a representation of handedness when right-handed individuals make responses with their left hand (Hayashi et al. 2008). To assess whether such representations were observable in our data, subsequent analyses assessed whether the degree of right-handedness (assessed with the EHI score) predicted activations when responding with the left hand. These analyses were separately assessed for both within- and inter-hemispheric transfer, and in both gray and white matter.

\section{(b) How does the degree of right-handedness predict activations during uni- manual responses with the left hand?-Figure 6 depicts significant clusters from the}


regression analyses of activations in gray matter for the within hemispheric (a) and the interhemispheric (b) conditions. Two broad effects are notable. First, regardless of condition, increased right-handedness only predicts decreased activations when responding with the left hand. These effects were observed in regions both within and outside core motor regions (statistical details are presented in Table 5). Second, the extent of this effect was more clearly marked for the IT than the within hemispheric transfer condition. In addition to the loci of activation presented in Figure 6, graphs depict mean activation values as a function of the degree of right handedness in selected peaks: In each of left M1 (i.e., ipsi-lateral to the responding hand) and cerebellum.

Regression effects were separately assessed in white matter. As seen in Figures $7 \mathrm{a}$ and $7 \mathrm{~b}$ the direction of these effects (decreased activations with increased right handedness) were identical to those observed in gray matter. Generally, the extent of these effects is similar across the within and inter-hemispheric conditions though some differences in the locations of loci are notable, particularly in the corpus callosum where the observed clusters were more extensive in the inter-hemispheric condition (Table 6). To highlight these differences, graphs depict mean activation values as a function of right-handedness in the corpus callosum peak for each of the conditions.

(b) How does the age predict activations during uni-manual responses with the left hand?-Figure 8 depicts significant clusters from the regression analyses of activations in gray matter for the within hemispheric (a) and the inter-hemispheric (b) conditions. Two broad effects are notable. First, regardless of condition, increased age predicts decreased activations when responding with the left hand, and secondly in gray matter these effects are more generally observed during the inter-hemispheric condition.

These effects were also probed in white matter (Figure 9, Table 8). As observed in Figure $9 \mathrm{~b}$, the age-related effects associated with the inter-hemispheric condition were more widely observed than with the within-hemispheric condition.

\section{Discussion}

This study was driven by multiple a priori and a posteriori motivations: 1) First, we were interested in whether the response hand in right handed subjects modulated activations during within- and inter-hemispheric transfer (assessed using the Poffenberger paradigm); 2) Second (and as a corollary of the primary aim), we were interested in whether these patterns could be identified not only in gray matter, but in circumscribed white matter tracts, quantified using a probabilistic atlas; 3 ) Third, after it emerged that right handed activations were slightly more prominent, particularly during inter-hemispheric transfer in both gray and white matter (Figures 4 and 5) we also explored if the degree of right-handedness (as estimated with the EHI) was represented in uni-manual responses with the left hand. This exploration was motivated by previous evidence suggestive of representations of righthandedness in when right-handers make responses with the left hand (Yalachkov et al. 2015), which suggests that inhibitory processes between bilateral motor regions may underlie hand dominance in right-handed individuals (Tzourio-Mazoyer et al. 2015). Finally, 
given prior evidence of structural and behavioral changes in middle adulthood, we assessed the effects of age on responses made with the non-dominant hand (Diwadkar et al. 2016).

We reprise our results: 1) Behavioral analyses indicated that responses with the right hand were faster (Figure 3) expectedly suggesting privileged responses for the dominant hand. 2) Analyses in gray matter during within and inter-hemispheric transfer conditions when responding with the right and left hand showed symmetric and asymmetric activations. As seen in Figure 4 and Tables 2 and 3, hand-related activations were observed in contra-lateral motor and ipsi-lateral cerebellar regions. These effects presumably reflect established representations of motor responses for each hand in right-handed subjects (that are irrespective of task-condition). Moreover, responses with the right hand resulted in privileged activations during the inter-hemispheric transfer condition (Figure 4b, Table 3). These were observed in regions that have been associated with motor pathways including the supplementary motor area associated with directed motor movements (Asemi et al. 2015; Grefkes et al. 2008), and heteromodal areas like the dorsolateral prefrontal cortex associated with executive control. Moreover, the extent of activated clusters were greater for the right hand in the IT condition (Table 3). 3) Functional activations in white matter while exploratory also indicated a particularly strong effect of response hand during interhemispheric transfer (Figure 5, Table 4). These privileged activations were observed in both transverse and longitudinal tracts. 4) The degree of right-handedness exerted strong effects on activations associated with left hand responses. These effects observed in gray and white matter, indicated that increased right-handedness was associated with decreased activation when responding with the left hand (Figures $6 \& 7$ ). 5) Finally, the extent of activations in the left hand decreased with participant age in both white and gray matter (Figures 8 and 9), with the effects particularly notable in the IT condition.

In the remainder of the paper we speculate on the relevance of our results for understanding both representations of handedness, visuo-motor integration and within and interhemispheric transfer, and the relationship between these fundamental organizational principles of brain networks. We also comment on the credibility of our observed fMRI activations in white matter, and particularly their functional relevance in study plausible network bases of task implementations (Gawryluk et al. 2014).

\section{Gray matter activations for each hand and transfer condition within the core and extended motor system}

Several loci were preferentially activated for either hand within each transfer condition. Several of these sites conform to previous meta-analyses of finger tapping studies (irrespective of task) in right-handed participants and can therefore be considered as unremarkable. These sites include contra-lateral M1 and supplementary motor area (SMA) (c.f., Figure 2a), and were symmetric across responses with either hand. Tables 2 and 3 highlight these symmetries. Symmetries were also observed in the ipsi-lateral cerebellum, and are somewhat notable given that the current task did not impose explicit chronometric demands, and that the cerebellum has been particularly implicated in finger movements involving externally or internally guided timing (Kawashima et al. 2000; Dreher and Grafman 2002). These effects imply a more general role for the cerebellum than previously 
suggested yet as these effects are consistently observed regardless of transfer condition, they are likely attributable to fundamental motor demands of each task.

Within the inter-hemispheric transfer condition, unique activation sites were observed for the right hand in the SMA and the dorsal prefrontal cortex and greater extent of activations were evident in the basal ganglia (putamen), the thalamus, and the dorsal anterior cingulate cortex. The effects in the thalamus and the putamen are notable given these regions' roles in sensori-motor pathways. The thalamus is an active relay center with complex functional interactions with the cortex during attention (Jagtap and Diwadkar 2016) and basic motor tasks (Lin et al. 2009). The basal ganglia are part of the extra-pyramidal motor system (Herrero et al. 2002), and receive inputs from multiple cortical regions, and from the thalamus. These inputs are projected to frontal regions, presumably in the service of higherorder motor control and planning (Haber and Calzavara 2009). A reasonable inference is that inter-hemispheric transfer of information evokes stronger representations in the extended motor pathway when responding with the right hand. The dynamic modulation by transfer demand may therefore reflect functional representations of right-handedness beyond the core motor circuit. This notion is compelling given evidence from experimental work and computational modeling that motor function can "vicariously" reorganize in response to lesions (Jaillard et al. 2005; Grecucci et al. 2008). Our results suggest that functional response properties are highly and systematically modifiable.

The regression analyses supplement some of these speculations. When responding with the left hand, the degree of right-handedness exerted substantial statistical effects during both within- and inter-hemispheric transfer, but the extent of the effects was particularly pronounced in the latter (Figure 6b). Significant loci beyond the core motor system were in concordance with the effects depicted in Figure 4. That is, when responding with the left hand, increased right-handedness predicted decreased responses in frontal, thalamic and basal ganglia regions. Moreover, regression analyses also revealed compelling effects in M1 and the cerebellum. Whereas responses with the left hand result in strongly privileged activations in contra-lateral M1 and ipsi-lateral cerebellum (Figure 4a and 4b), decreased responses in contra-lateral (right) M1 were predicted by increased right-handedness. As has been shown in studies in multiple modalities, finger responses with the dominant right hand result in strong deactivations of ipsi-lateral (i.e., right) M1 (Tzourio-Mazoyer et al. 2015) because in right-handed individuals transcallosal inhibition from the ipsi-lateral (i.e., right) M1 underpins laterality (Grefkes et al. 2008; Hayashi et al. 2008). By extension, the degree of right-handedness (i.e. laterality) should predict decreased activation of right M1 when responding with the left hand. Indeed, these effects were very strongly associated with the inter-hemispheric transfer condition (Figure 6b; red insets), and constitute evidence for a modified version of the idea that inhibitory mechanisms underlie right-handed dominance.

In addition, substantial effects of the degree of right-handedness were observed in both ipsilateral (i.e. left) M1 and contra-lateral (i.e., right) cerebellum, that is homologues of right M1 and left cerebellum, regions typically activated in left hand responses (see also Figure 4). These effects were generic (across transfer condition), and may reflect an organizational characteristic of the motor system in right-handers, that to our knowledge is previously unexplored. 
Age itself exerted substantial statistical effects on gray matter activations in responding with the non-dominant hand (Figure 8). As seen, largely negative correlations were observed, indicating that increased age in middle adulthood (Diwadkar et al. 2016)lead to a decrease in the engagement of brain regions during both within and inter-hemispheric transfer.

Moreover, the cluster sizes were larger in the IT condition (see Table 7), consistent with the previous analyses, again evidence that IT-related representations are privileged in some way. Cross-sectional assessments of life-span changes are intrinsically challenging to interpret (Raz and Lindenberger 2011) because individual variability itself changes across periods of the life-span and can statistically overwhelm meaningful estimate of true changes. Moreover, a source of fMRI activations lies in the complexity of task-induced modulation (Logothetis 2008) that may only loosely be constrained by the brain's structural properties (Park and Friston 2013; Price and Friston 2005). Yet that these statistical associations are asymmetric (greater for IT than Within). If age acts to decrease the integrity of white matter (Serbruyns et al. 2016), it is plausible that the more demanding IT condition will evoke a stronger representation of the functional effect of this decrease. In any case, the study of life-span changes is a field into itself and cannot be meaningful addressed in a parenthetical manner. A more detailed assessment of these questions is ongoing.

\section{White matter activations for each hand and transfer condition}

White matter activations in the context of fMRI data have been the subject of debate, but evidence increasingly suggests that under appropriately constrained experimental conditions, these effects reflect properties of the brain's functional network architecture (Gawryluk et al. 2014). White matter activations have been independently reported in several studies and across tasks, recruited samples, and acquisition techniques (echo-planar or spin-echo imaging) (Gawryluk et al. 2011a; Gawryluk et al. 2011b; Gawryluk et al. 2009; Tettamanti et al. 2002; Yarkoni et al. 2009; Weber et al. 2005; Stroman and Ryner 2001)(a full treatment is beyond our scope). fMRI activations have been shown to follow fiber tracts within structures such as the corpus callosum, evidence that these activations constitute a functional signature of the underlying connectivity of the brain (Mazerolle et al. 2010). These robust results are observed despite sparse vascularization in white matter that can affect the amplitude and spatial resolution of the hemodynamic response, and reduced energy consumption in white (compared to grey) matter that may preclude reliable detection of BOLD (Logothetis and Wandell 2004).

Our approach toward characterizing these effects has been motivated by the specific task demands (that engage substantial intra- and inter-hemispheric integration of signals), and was conservatively implemented. Thus, after applying a relatively narrow smoothing filter during the pre-processing of data, we only categorized as reliable, activations that lay within probabilistically demarcated white matter tracts. The relatively conservative nature of this approach is highlighted when our tract-based mask is considered relative to a generic white matter mask that serves as the primary overlay template in Figures 5,7 and 9.

With these caveats, our white matter activations provide evidence of condition-specific effects of response hand. Specifically, the effects in Figure 5b provide evidence that white matter activations are more strongly modulated by response hand under inter-hemispheric 
transfer (no privileged activations are observed for the left hand). These effects are notable because they provide stronger specificity than comparable results observed in gray matter (Figure 4b), and constitute compelling evidence for an interaction between response hand and IT in right-handers. The analysis of handedness was similarly compelling. Within the inter-hemispheric transfer condition, no privileged activations were observed for left-hand responses in white matter (Figure 5b); however increased right-handedness strongly predicted decreased activations in white matter during left-handed responses (Figure 7b). Some of these clusters were in spatial concordance with those observed when subjects responded with their right hand. Whereas it is challenging to interpret the import of these loci in longitudinal tracts, the fact that several clusters were observed in callosal regions including the genu, body and the splenium is notable.

Trans-callosal motor signals and inter-hemispheric communication are central to basic motor behavior (Geffen et al. 1994). In the context of inter-hemispheric transfer tasks, transfer costs appear specifically when responses are made with hands, but do not appear when other effectors (e.g., lower limbs) are used (Berlucchi et al. 1995). These documented behavioral effects imply that inter-hemispheric communication is strongly associated with the motor system of the hand though these relationships had not been subsequently probed in vivo. Lesion studies in primates have provided further evidence of the structural bases of these effects wherein callosal lesions preempt or depress the learning of inter-manual motor transfer learning in macaques (Eacott and Gaffan 1990), and the critical role of the corpus callosum in the integration of inter-hemispheric signals in visuo-motor tasks is well established (Schulte and Muller-Oehring 2010). Furthermore callosal recruitment is dynamically mediated by response characteristics during uni-manual responding (Serrien et al. 2006). Therefore, evidence that callosal activations when responding with the left hand are predicted by increased right-handedness is consistent with a dynamic and inhibitory role for the structure in sub-serving right-handedness (Bloom and Hynd 2005). However, these observed effects are neutral on the question of excitatory (Gazzaniga 2000) or inhibitory models of callosal (Allison et al. 2000) or inter-hemispheric motor function (Kobayashi et al. 2003). These models have largely relied on response competition, but our design specifically eliminated competitive response choice (by blocking response hand). Therefore, in general our effects are more likely to reflect organizational aspects of motor function, rather than competitive characteristics of interactions across motor systems.

The physiological and cellular bases of these effects are challenging to interpret, specifically because as previously noted, fMRI signals are only broadly constrained by the brain's structural properties and must be considered highly modifiable under task-characteristics (Park and Friston 2013; Huang and Ding 2016). This means that the observed effects in white matter might relate to structural or physiological characteristics of white matter pathways in right handers that are unique (though our experimental design did not include left handed participants). Moreover, the functional properties of white matter (indexed with fMRI) are modified by insults such as traumatic brain injury; post injury, fMRI responses in longitudinal fiber tracts such as the superior longitudinal fasciculus and the anterior capsule are reduced during a visual tracking task (Astafiev et al. 2015) suggesting that modification to the cellular characteristics of white matter micro-structure are reflected in fMRI estimated activation. In general, inter-hemispheric transmission plays a critical recovery role in post- 
stroke rehabilitation, such that intact inter-hemispheric commissures facilitate the intact hemisphere supplementing brain function in areas of the damaged hemisphere (Bartolomeo and Thiebaut de Schotten 2016).

There is stronger evidence of left- than right-neglect in post-stroke studies, suggestive of recovery related impairments in right handers (compared to left handers) related to mechanisms of spatial bias (Bareham et al. 2015). This implies that underlying physiology of white matter pathways in right handers may be less "plastic." Such an interpretation is consistent with the evidence in white matter pathways, right-hand related responses in right handers evoke stronger representations, than do left-hand responses. Recent evidence from in vivo diffusion tensor imaging is consistent with such an interpretation: White matter micro-structure is characterized by significantly lower fractional anisotropies in rightcompared to left-handers (McKay et al. 2017), indicative of more constrained organization of structural pathways. It is open question whether a study of left handers will provide enantiomorphic representations to those observed in Figures 6 and 7. We are presently investigating this intriguing direction.

These results provide evidence of previously un-assessed representations of (right) handedness and correlates of within- and inter-hemisphere transfer revealed with the Poffenberger paradigm. We note that our focus on representations of right-handedness and its effects preclude us from investigating hemispheric differences associated with right- or left-handedness (Amunts et al. 2000; Amunts et al. 1996), restricting the plausible generalizations of these data for an understanding of motor organization. Moreover, it was precisely this focus that also oriented us towards more conservative criteria for righthandedness based on the EHI. The results are also agnostic with respective to the network based mechanisms underlying IT. For example effective connectivity analyses have provided highly compelling evidence of complex patterns of inter-hemispheric network interactions and integrations underlying uni- and bi-manual movements (Grefkes et al. 2008) and visual judgments (Stephan et al. 2007), providing the promise of allowing us to better clarify the bases of the observed asymmetries in activation.

Against these limitations, we reiterate that our results while complex, retain several novel aspects. The response hand used by right-handers during this task, and the degree of righthandedness exert substantial signatures during within-, and particularly inter-hemispheric transfer. These effects suggest substantial interactions between the organization of handedness and the functional "flow" of information within and between the hemispheres. These interactions may depend on the systematic sensori-motor organization of motor function (an organization that is relied on by the Poffenberger paradigm and other interhemispheric transfer tasks for assessing within- and inter-hemispheric effects) and its dynamic expression under task conditions with circumscribed demands. These studies further highlight the complex dialectic between structure-function relationships in the brain (Park and Friston 2013; Price and Friston 2005) even under the simplest of task conditions. Moreover, they suggest a simple framework for interrogating the functional integrity of macroscopic brain networks in multiple neuropsychiatric and neurological populations. 


\section{Acknowledgments}

This study was partly supported from grants from Fondazione CariVerona (Obiettivo di ricerca A9 del progetto di Neuroscience "Disabilità cognitiva e comportamentale nelle demenze e nelle psicosi") to PB and MR and from the Ministry of Health to PB (GR-2010-2319022), and MB (GR-2010-2316745). VAD was supported by a Cooperint Visiting Professorship from the University of Verona, a Career Development Chair from Wayne State University, the Thomas Gershenson Distinguished Faculty Fellowship from Wayne State University, the Lyckaki-Young Fund from the State of Michigan, the Prechter Family Bipolar Foundation, the Children's Hospital of Michigan Foundation and the Cohen Neuroscience Endowment. We thank Alberto Beltramello for helping in MRI acquisition and data management and Gianluca Rambaldelli for help in data processing.

\section{References}

Aboitiz F, Lopez J, Montiel J. Long distance communication in the human brain: timing constraints for inter-hemispheric synchrony and the origin of brain lateralization. Biol Res. 2003; 36(1):89-99. [PubMed: 12795208]

Allison JD, Meador KJ, Loring DW, Figueroa RE, Wright JC. Functional MRI cerebral activation and deactivation during finger movement. Neurology. 2000; 54(1):135-142. [PubMed: 10636139]

Amaro E Jr, Barker GJ. Study design in fMRI: Basic principles. Brain Cogn. 2006; 60(3):220-232. [PubMed: 16427175]

Amunts K, Jancke L, Mohlberg H, Steinmetz H, Zilles K. Interhemispheric asymmetry of the human motor cortex related to handedness and gender. [Clinical Trial Research Support, Non-U.S. Gov't]. Neuropsychologia. 2000; 38(3):304-312. [PubMed: 10678696]

Amunts K, Schlaug G, Schleicher A, Steinmetz H, Dabringhaus A, Roland PE, et al. Asymmetry in the human motor cortex and handedness. [Research Support, Non-U.S. Gov't]. Neuroimage. 1996; 4(3 Pt 1):216-222. DOI: 10.1006/nimg.1996.0073 [PubMed: 9345512]

Asemi A, Ramaseshan K, Burgess A, Diwadkar VA, Bressler SL. Dorsal anterior cingulate cortex modulates supplementary motor area in coordinated unimanual motor behavior. Front Hum Neurosci. 2015; 9:309.doi: 10.3389/fnhum.2015.00309 [PubMed: 26089783]

Ashburner J, Friston KJ. Unified segmentation. Neuroimage. 2005; 26(3):839-851. doi:S1053-8119(05)00110-2 [pii] 10.1016/j.neuroimage.2005.02.018. [PubMed: 15955494]

Astafiev SV, Shulman GL, Metcalf NV, Rengachary J, MacDonald CL, Harrington DL, et al. Abnormal White Matter Blood-Oxygen-Level-Dependent Signals in Chronic Mild Traumatic Brain Injury. J Neurotrauma. 2015; 32(16):1254-1271. DOI: 10.1089/neu.2014.3547 [PubMed: 25758167]

Bareham CA, Bekinschtein TA, Scott SK, Manly T. Does left-handedness confer resistance to spatial bias? Sci Rep. 2015; 5:9162.doi: 10.1038/srep09162 [PubMed: 25781078]

Bartolomeo P, Thiebaut de Schotten M. Let thy left brain know what thy right brain doeth: Interhemispheric compensation of functional deficits after brain damage. Neuropsychologia. 2016; 93(Pt B):407-412. DOI: 10.1016/j.neuropsychologia.2016.06.016 [PubMed: 27312744]

Berlucchi G, Aglioti S, Marzi CA, Tassinari G. Corpus callosum and simple visuomotor integration. [Review]. Neuropsychologia. 1995; 33(8):923-936. [PubMed: 8524454]

Bloom JS, Hynd GW. The role of the corpus callosum in interhemispheric transfer of information: excitation or inhibition? [Research Support, N.I.H., Extramural Review]. Neuropsychol Rev. 2005; 15(2):59-71. DOI: 10.1007/s11065-005-6252-y [PubMed: 16211466]

Byrne M, Clafferty RA, Cosway R, Grant E, Hodges A, Lawrie SM, et al. Measurement of lateral preferences and schizophrenia: results of the Edinburgh High-Risk Study and methodological issues. [Research Support, Non-U.S. Gov't]. Psychiatry Res. 2004; 125(3):205-217. DOI: 10.1016/j.psychres.2004.01.001 [PubMed: 15051181]

Dale AM. Optimal experimental design for event-related fMRI. [Research Support, Non-U.S. Gov't Research Support, U.S. Gov’t, P.H.S.]. Hum Brain Mapp. 1999; 8(2-3):109-114. [PubMed: 10524601]

Diwadkar VA, Asemi A, Burgess A, Chowdury A, Bressler SL. Potentiation of motor sub-networks for motor control but not working memory: Interaction of AACC and SMA revealed by resting-state 
directed functional connectivity. PLoS ONE. 2017; 12(3):e0172531.doi: 10.1371/journal.pone. 0172531 [PubMed: 28278267]

Diwadkar VA, Bellani M, Ahmed R, Dusi N, Rambaldelli G, Perlini C, et al. Chronological age and its impact on associative learning proficiency and brain structure in middle adulthood. Behav Brain Res. 2016; 297:329-337. DOI: 10.1016/j.bbr.2015.10.016 [PubMed: 26462573]

Dragovic M. Categorization and validation of handedness using latent class analyses. Acta Neuropsychiatrica. 2004; 16:212-218. [PubMed: 26984309]

Dreher JC, Grafman J. The roles of the cerebellum and basal ganglia in timing and error prediction. Eur J Neurosci. 2002; 16(8):1609-1619. doi:2212 [pii]. [PubMed: 12405975]

Eacott MJ, Gaffan D. Interhemispheric transfer of visuomotor conditional learning via the anterior corpus callosum of monkeys. [Research Support, Non-U.S. Gov't]. Behav Brain Res. 1990; 38(2): 109-116. [PubMed: 2363832]

Fling BW, Seidler RD. Fundamental differences in callosal structure, neurophysiologic function, and bimanual control in young and older adults. [Comparative Study Research Support, N.I.H., Extramural]. Cereb Cortex. 2012; 22(11):2643-2652. DOI: 10.1093/cercor/bhr349 [PubMed: 22166764]

Friedman A, Burgess A, Ramaseshan K, Easter P, Khatib D, Chowdury A, et al. Brain network dysfunction in obsessive-compulsive disorder induced by simple uni-manual behavior: The role of the dorsal anterior cingulate cortex. Psychiatry Res Neuroimaging. 2017; 260:6-15. [PubMed: 27992792]

Friston KJ, Holmes AP, Worsely KJ, Poline JB, Frith CD, J FRS. Statistical parametric maps in functional imaging: a general approach. Hum Brain Mapp. 1995; 2:189-210.

Gawryluk JR, Brewer KD, Beyea SD, D’Arcy RC. Optimizing the detection of white matter fMRI using asymmetric spin echo spiral. [Evaluation Studies Research Support, Non-U.S. Gov't]. Neuroimage. 2009; 45(1):83-88. DOI: 10.1016/j.neuroimage.2008.11.005 [PubMed: 19084071]

Gawryluk JR, D’Arcy RC, Mazerolle EL, Brewer KD, Beyea SD. Functional mapping in the corpus callosum: a 4T fMRI study of white matter. [Research Support, Non-U.S. Gov't]. Neuroimage. 2011a; 54(1):10-15. DOI: 10.1016/j.neuroimage.2010.07.028 [PubMed: 20643213]

Gawryluk JR, Mazerolle EL, Brewer KD, Beyea SD, D'Arcy RC. Investigation of fMRI activation in the internal capsule. [Research Support, Non-U.S. Gov't]. BMC Neurosci. 2011b; 12:56.doi: 10.1186/1471-2202-12-56 [PubMed: 21672250]

Gawryluk JR, Mazerolle EL, D’Arcy RC. Does functional MRI detect activation in white matter? A review of emerging evidence, issues, and future directions. [Review]. Front Neurosci. 2014; 8:239.doi: 10.3389/fnins.2014.00239 [PubMed: 25152709]

Gazzaniga MS. Organization of the human brain. Science. 1989; 245(4921):947-952. [PubMed: 2672334]

Gazzaniga MS. Cerebral specialization and interhemispheric communication: does the corpus callosum enable the human condition? Brain. 2000; 123(Pt 7):1293-1326. [PubMed: 10869045]

Geffen GM, Jones DL, Geffen LB. Interhemispheric control of manual motor activity. [Research Support, Non-U.S. Gov't Review]. Behav Brain Res. 1994; 64(1-2):131-140. [PubMed: 7840879]

Grecucci A, Crescentini C, Siugzdaite R. Vicarious function in the motor cortex. A computational investigation. Neurosci Lett. 2008; 434(2):185-190. DOI: 10.1016/j.neulet.2008.01.059 [PubMed: 18291583]

Grefkes C, Eickhoff SB, Nowak DA, Dafotakis M, Fink GR. Dynamic intra- and interhemispheric interactions during unilateral and bilateral hand movements assessed with fMRI and DCM. Neuroimage. 2008; 41(4):1382-1394. doi:S1053-8119(08)00283-8 [pii] 10.1016/j.neuroimage. 2008.03.048. [PubMed: 18486490]

Haber SN, Calzavara R. The cortico-basal ganglia integrative network: the role of the thalamus. Brain Res Bull. 2009; 78(2-3):69-74. doi:S0361-9230(08)00342-0 [pii] 10.1016/j.brainresbull. 2008.09.013. [PubMed: 18950692]

Hayashi MJ, Saito DN, Aramaki Y, Asai T, Fujibayashi Y, Sadato N. Hemispheric asymmetry of frequency-dependent suppression in the ipsilateral primary motor cortex during finger movement: a functional magnetic resonance imaging study. [Research Support, Non-U.S. Gov't]. Cereb Cortex. 2008; 18(12):2932-2940. DOI: 10.1093/cercor/bhn053 [PubMed: 18413350] 
Hellige JB. Hemispheric asymmetry for visual information processing. [Research Support, U.S. Gov't, Non-P.H.S. Review]. Acta Neurobiol Exp (Wars). 1996; 56(1):485-497. [PubMed: 8787209]

Herrero MT, Barcia C, Navarro JM. Functional anatomy of thalamus and basal ganglia. Childs Nerv Syst. 2002; 18(8):386-404. DOI: 10.1007/s00381-002-0604-1 [PubMed: 12192499]

Hua K, Zhang J, Wakana S, Jiang H, Li X, Reich DS, et al. Tract probability maps in stereotaxic spaces: analyses of white matter anatomy and tract-specific quantification. [Research Support, N.I.H., Extramural Research Support, Non-U.S. Gov't]. Neuroimage. 2008; 39(1):336-347. DOI: 10.1016/j.neuroimage.2007.07.053 [PubMed: 17931890]

Huang H, Ding M. Linking Functional Connectivity and Structural Connectivity Quantitatively: A Comparison of Methods. Brain Connect. 2016; 6(2):99-108. DOI: 10.1089/brain.2015.0382 [PubMed: 26598788]

Jagtap P, Diwadkar VA. Effective connectivity of ascending and descending frontalthalamic pathways during sustained attention: Complex brain network interactions in adolescence. Hum Brain Mapp. 2016; doi: 10.1002/hbm.23196

Jaillard A, Martin CD, Garambois K, Lebas JF, Hommel M. Vicarious function within the human primary motor cortex? A longitudinal fMRI stroke study. [Research Support, Non-U.S. Gov't]. Brain. 2005; 128(Pt 5):1122-1138. DOI: 10.1093/brain/awh456 [PubMed: 15728652]

JeannerodM. The neural and behavioural organization of goal directed movementsOxford, United Kingdom: Oxford University Press; 1988

Josephs O, Henson RN. Event-related functional magnetic resonance imaging: modelling, inference and optimization. Philos Trans R Soc Lond B Biol Sci. 1999; 354(1387):1215-1228. [PubMed: 10466147]

Kawashima R, Okuda J, Umetsu A, Sugiura M, Inoue K, Suzuki K, et al. Human cerebellum plays an important role in memory-timed finger movement: an fMRI study. J Neurophysiol. 2000; 83(2): 1079-1087. [PubMed: 10669519]

Kim SG, Ashe J, Hendrich K, Ellermann JM, Merkle H, Ugurbil K, et al. Functional magnetic resonance imaging of motor cortex: hemispheric asymmetry and handedness. [Research Support, Non-U.S. Gov't Research Support, U.S. Gov’t, P.H.S.]. Science. 1993; 261(5121):615-617. [PubMed: 8342027]

Kobayashi M, Hutchinson S, Schlaug G, Pascual-Leone A. Ipsilateral motor cortex activation on functional magnetic resonance imaging during unilateral hand movements is related to interhemispheric interactions. [Research Support, Non-U.S. Gov't Research Support, U.S. Gov't, P.H.S.]. Neuroimage. 2003; 20(4):2259-2270. [PubMed: 14683727]

Lin FH, Agnew JA, Belliveau JW, Zeffiro TA. Functional and effective connectivity of visuomotor control systems demonstrated using generalized partial least squares and structural equation modeling. [Research Support, N.I.H., Extramural Research Support, Non-U.S. Gov't]. Hum Brain Mapp. 2009; 30(7):2232-2251. DOI: 10.1002/hbm.20664 [PubMed: 19288462]

Liu TT. Efficiency, power, and entropy in event-related fMRI with multiple trial types. Part II: design of experiments. [Comparative Study Research Support, Non-U.S. Gov't Research Support, U.S. Gov't, Non-P.H.S.]. Neuroimage. 2004; 21(1):401-413. [PubMed: 14741677]

Logothetis NK. What we can do and what we cannot do with fMRI. Nature. 2008; 453(7197):869878. doi:nature06976 [pii] 10.1038/nature06976. [PubMed: 18548064]

Logothetis NK, Wandell BA. Interpreting the BOLD signal. Annu Rev Physiol. 2004; 66:735-769. [PubMed: 14977420]

Maldjian JA, Laurienti PJ, Kraft RA, Burdette JH. An automated method for neuroanatomic and cytoarchitectonic atlas-based interrogation of fMRI data sets. Neuroimage. 2003; 19(3):12331239. [PubMed: 12880848]

Martuzzi R, Murray MM, Maeder PP, Fornari E, Thiran J, Clarke S, et al. Visuo-motor pathways in humans revealed by event-related fMRI. [Research Support, Non-U.S. Gov't]. Exp Brain Res. 2006; 170(4):472-487. DOI: 10.1007/s00221-005-0232-6 [PubMed: 16307262]

Marzi CA. The Poffenberger paradigm: a first, simple, behavioural tool to study interhemispheric transmission in humans. [Biography Historical Article]. Brain Res Bull. 1999; 50(5-6):421-422. [PubMed: 10643464] 
Marzi CA, Bisiacchi P, Nicoletti R. Is interhemispheric transfer of visuomotor information asymmetric? Evidence from a meta-analysis. [Meta-Analysis]. Neuropsychologia. 1991; 29(12): 1163-1177. [PubMed: 1838793]

Mazerolle EL, Beyea SD, Gawryluk JR, Brewer KD, Bowen CV, D'Arcy RC. Confirming white matter fMRI activation in the corpus callosum: co-localization with DTI tractography. [Research Support, Non-U.S. Gov't]. Neuroimage. 2010; 50(2):616-621. DOI: 10.1016/j.neuroimage.2009.12.102 [PubMed: 20053383]

Mazerolle EL, D’Arcy RC, Beyea SD. Detecting functional magnetic resonance imaging activation in white matter: interhemispheric transfer across the corpus callosum. [Clinical Trial Research Support, Non-U.S. Gov't]. BMC Neurosci. 2008; 9:84.doi: 10.1186/1471-2202-9-84 [PubMed: 18789154]

McKay NS, Iwabuchi SJ, Haberling IS, Corballis MC, Kirk IJ. Atypical white matter microstructure in left-handed individuals. Laterality. 2017; 22(3):257-267. DOI: 10.1080/1357650X.2016.1175469 [PubMed: 27119972]

Mooshagian E, Iacoboni M, Zaidel E. Spatial attention and interhemispheric visuomotor integration in the absence of the corpus callosum. [Case Reports Research Support, N.I.H., Extramural]. Neuropsychologia. 2009; 47(3):933-937. DOI: 10.1016/j.neuropsychologia.2008.12.005 [PubMed: 19124029]

Nachev P, Wydell H, O’Neill K, Husain M, Kennard C. The role of the pre-supplementary motor area in the control of action. [Case Reports Research Support, Non-U.S. Gov't]. Neuroimage. 2007; (36 Suppl 2):T155-163. DOI: 10.1016/j.neuroimage.2007.03.034 [PubMed: 17499162]

Oldfield RC. The assessment and analysis of handedness: the Edinburgh inventory. Neuropsychologia. 1971; 9(1):97-113. [PubMed: 5146491]

Park HJ, Friston K. Structural and functional brain networks: from connections to cognition. [Research Support, Non-U.S. Gov't Review]. Science. 2013; 342(6158):1238411.doi: 10.1126/science. 1238411 [PubMed: 24179229]

Poffenberger AT. Reaction time to retinal stimulation with special reference to the time lost in conduction through nervous centers. Arch Psychol. 1912; 23:1-73.

Price CJ, Friston KJ. Functional ontologies for cognition: The systematic definition of structure and function. Cogn Neuropsychol. 2005; 22(3):262-275. DOI: 10.1080/02643290442000095 [PubMed: 21038249]

Raz N, Lindenberger U. Only time will tell: cross-sectional studies offer no solution to the age-braincognition triangle: comment on Salthouse (2011). [Comment Research Support, N.I.H., Extramural]. Psychol Bull. 2011; 137(5):790-795. DOI: 10.1037/a0024503 [PubMed: 21859179]

Savazzi S, Fabri M, Rubboli G, Paggi A, Tassinari CA, Marzi CA. Interhemispheric transfer following callosotomy in humans: role of the superior colliculus. [Research Support, Non-U.S. Gov't]. Neuropsychologia. 2007; 45(11):2417-2427. DOI: 10.1016/j.neuropsychologia.2007.04.002 [PubMed: 17509625]

Schulte T, Muller-Oehring EM. Contribution of callosal connections to the interhemispheric integration of visuomotor and cognitive processes. [Research Support, N.I.H., Extramural Review]. Neuropsychol Rev. 2010; 20(2):174-190. DOI: 10.1007/s11065-010-9130-1 [PubMed: 20411431]

Schulte T, Sullivan EV, Muller-Oehring EM, Adalsteinsson E, Pfefferbaum A. Corpus Callosal Microstructural Integrity Influences Interhemispheric Processing: A Diffusion Tensor Imaging Study. Cereb Cortex. 2005

Serbruyns L, Leunissen I, van Ruitenbeek P, Pauwels L, Caeyenberghs K, Solesio-Jofre E, et al. Alterations in brain white matter contributing to age-related slowing of task switching performance: The role of radial diffusivity and magnetization transfer ratio. Hum Brain Mapp. 2016; 37(11):4084-4098. DOI: 10.1002/hbm.23297 [PubMed: 27571231]

Serrien DJ, Ivry RB, Swinnen SP. Dynamics of hemispheric specialization and integration in the context of motor control. [Research Support, N.I.H., Extramural Research Support, Non-U.S. Gov't]. Nat Rev Neurosci. 2006; 7(2):160-166. DOI: 10.1038/nrn1849 [PubMed: 16429125] 
Stephan KE, Marshall JC, Penny WD, Friston KJ, Fink GR. Interhemispheric integration of visual processing during task-driven lateralization. J Neurosci. 2007; 27(13):3512-3522. doi:27/13/3512 [pii] 10.1523/JNEUROSCI.4766-06.2007. [PubMed: 17392467]

Stroman PW, Ryner LN. Functional MRI of motor and sensory activation in the human spinal cord. Magn Reson Imaging. 2001; 19(1):27-32. [PubMed: 11295343]

Tettamanti M, Paulesu E, Scifo P, Maravita A, Fazio F, Perani D, et al. Interhemispheric transmission of visuomotor information in humans: fMRI evidence. J Neurophysiol. 2002; 88(2):1051-1058. [PubMed: 12163553]

Toga AW, Thompson PM. Mapping brain asymmetry. [Research Support, U.S. Gov't, P.H.S. Review]. Nat Rev Neurosci. 2003; 4(1):37-48. DOI: 10.1038/nrn1009 [PubMed: 12511860]

Tootell RB, Mendola JD, Hadjikhani NK, Liu AK, Dale AM. The representation of the ipsilateral visual field in human cerebral cortex. [Research Support, Non-U.S. Gov't Research Support, U.S. Gov't, P.H.S. Review]. Proc Natl Acad Sci U S A. 1998; 95(3):818-824. [PubMed: 9448246]

Tzourio-Mazoyer N, Landeau B, Papathanassiou D, Crivello F, Etard O, Delcroix N, et al. Automated anatomical labeling of activations in SPM using a macroscopic anatomical parcellation of the MNI MRI single-subject brain. Neuroimage. 2002; 15(1):273-289. [PubMed: 11771995]

Tzourio-Mazoyer N, Petit L, Zago L, Crivello F, Vinuesa N, Joliot M, et al. Between-hand difference in ipsilateral deactivation is associated with hand lateralization: fMRI mapping of 284 volunteers balanced for handedness. Front Hum Neurosci. 2015; 9:5.doi: 10.3389/fnhum.2015.00005 [PubMed: 25705184]

van den Berg FE, Swinnen SP, Wenderoth N. Involvement of the primary motor cortex in controlling movements executed with the ipsilateral hand differs between left- and right-handers. [Research Support, Non-U.S. Gov't]. J Cogn Neurosci. 2011; 23(11):3456-3469. DOI: 10.1162/ jocn_a_00018 [PubMed: 21452954]

Weber B, Treyer V, Oberholzer N, Jaermann T, Boesiger P, Brugger P, et al. Attention and interhemispheric transfer: a behavioral and fMRI study. [Comparative Study]. J Cogn Neurosci. 2005; 17(1):113-123. DOI: 10.1162/0898929052880002 [PubMed: 15701243]

Yalachkov Y, Kaiser J, Doehrmann O, Naumer MJ. Enhanced visuo-haptic integration for the nondominant hand. [Research Support, Non-U.S. Gov't]. Brain Res. 2015; 1614:75-85. DOI: 10.1016/j.brainres.2015.04.020 [PubMed: 25911582]

Yarkoni T, Barch DM, Gray JR, Conturo TE, Braver TS. BOLD correlates of trial-by-trial reaction time variability in gray and white matter: a multi-study fMRI analysis. PLoS ONE. 2009; 4(1):e4257.doi: 10.1371/journal.pone.0004257 [PubMed: 19165335]

ZaidelE, , IacoboniM, editorsThe parallel brain: The cognitive neuroscience of the corpus callosumCambridge, MA: MIT Press; 2003

Zhang Y, Zhang J, Oishi K, Faria AV, Jiang H, Li X, et al. Atlas-guided tract reconstruction for automated and comprehensive examination of the white matter anatomy. [Research Support, N.I.H., Extramural]. Neuroimage. 2010; 52(4):1289-1301. DOI: 10.1016/j.neuroimage. 2010.05.049 [PubMed: 20570617] 



(1)

1

1

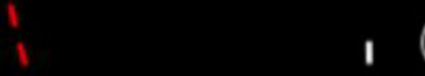

롤

Figure 1.

The figure provides a schematic depiction of the uni-manual Poffenberger paradigm (when responding with the right hand). (a) During the within-hemispheric condition, presenting the probe in the right visual field stimulates left V1, that is the hemisphere ipsi-lateral to the responding motor cortex. (b) During the inter-hemispheric transfer (IT) condition, presentation of the probe in the left visual field stimulates the right primary visual cortex (V1). The responding motor cortex is in the opposite hemisphere. 




Figure 2.

The dark shaded area (top right) in the depicted heat map signifies subjects classified as strongly right handed based on study criteria. The scoring criterion resulted in a relatively conservative classification ( $\geq 14$ classified as right-handed), relative to the traditional scoring in the Edinburgh handedness inventory ( $\geq 40$ classified as right handed) in a densely occupied region of the heat map. Lightly shaded regions depicted right-handedness classification based on the traditional scoring of the Edinburgh handedness inventory (lower right) and employed criteria (upper left). 


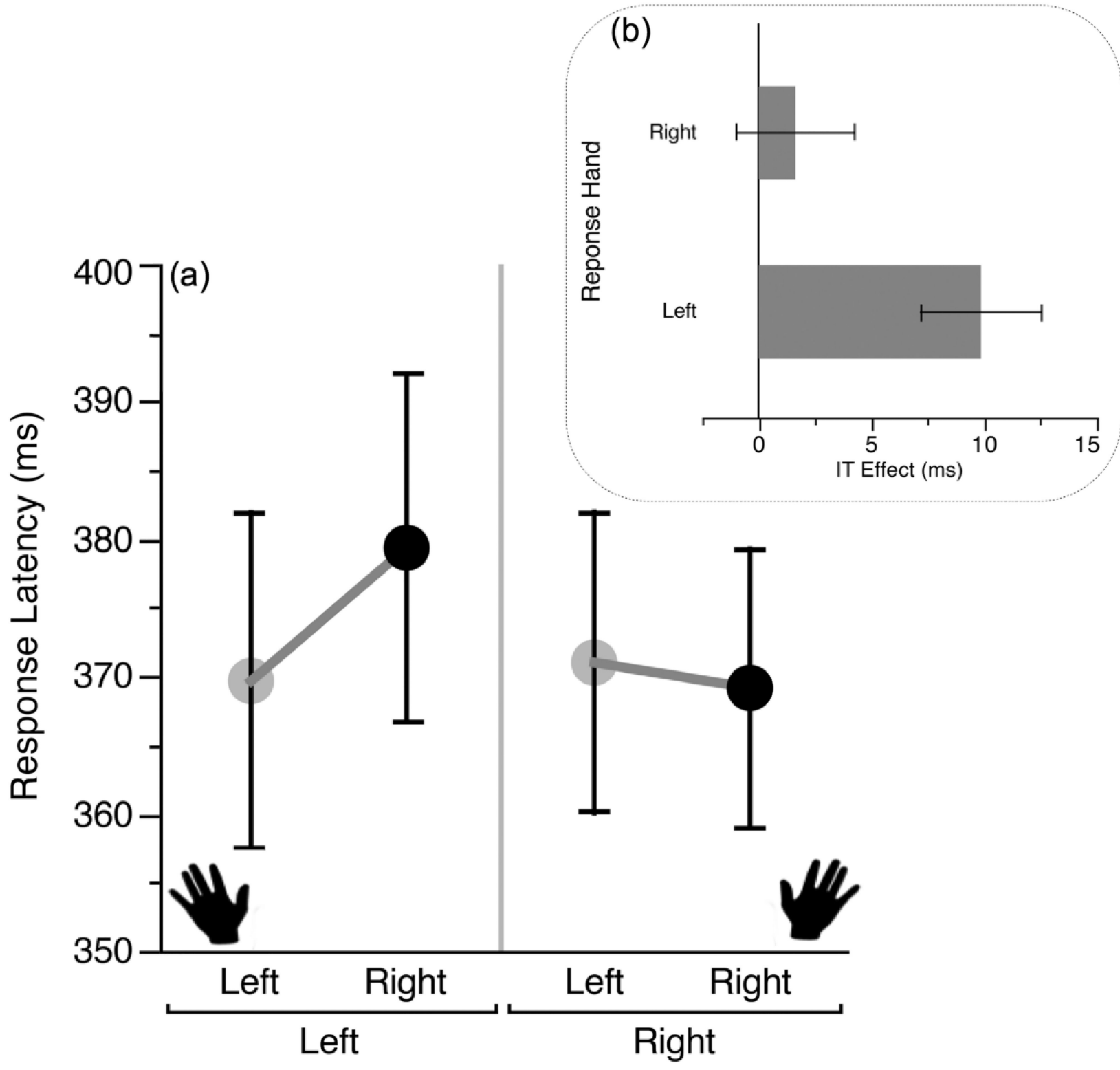

\section{Visual Field (within Response Hand)}

Figure 3.

(a) Mean response latencies as a function of response hand (Left or Right) and condition (Intra or Within) are depicted. The inset (b) depicts IT times (Intra - Within conditions) for each hand. A significant IT response latency effect was observed for the left hand (see text for details). Error bars are \pm sd. 


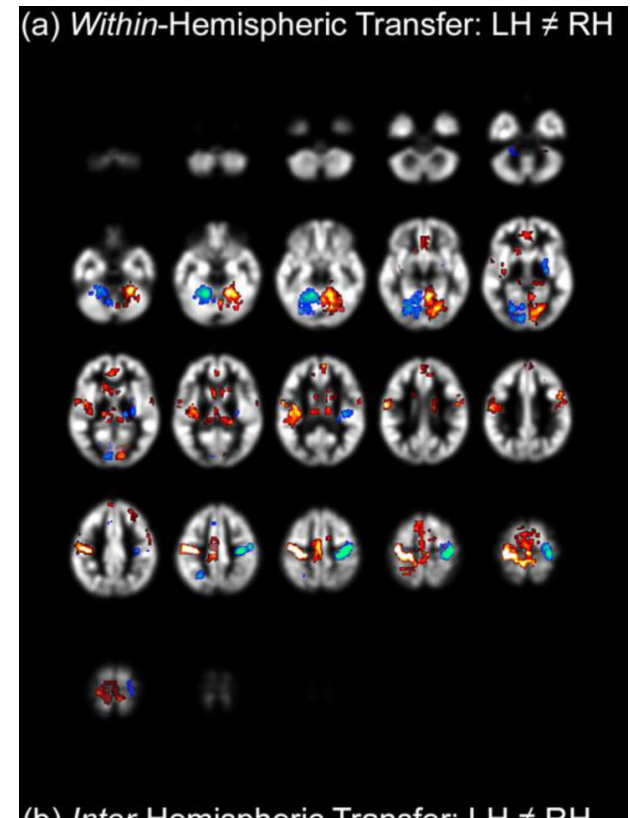

(b) Inter-Hemispheric Transfer: $\mathrm{LH} \neq \mathrm{RH}$

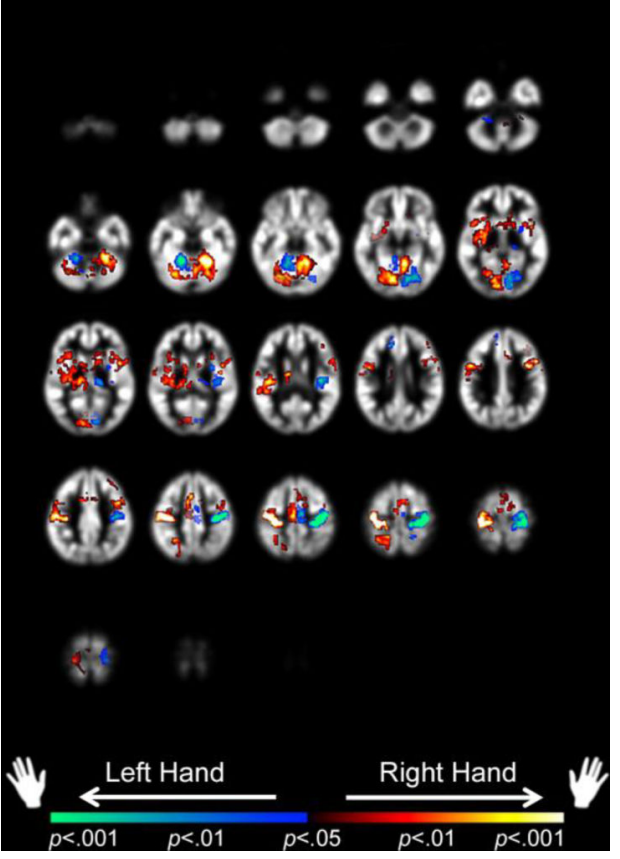

Figure 4.

Uni-manual effects in gray matter are depicted for each of the experimental conditions: a) Within-hemispheric transfer and b) Inter-hemispheric transfer. Warm colors denote increased activation for the dominant right hand; cool colors denote increased activation for the nondominant left hand. Labels are intentionally withheld from the images to reduce clutter, but detailed statistical information and loci are presented in Tables 2 and 3 respectively. As can be seen, the relative differences between uni-manual responses are strongly evident in the dACC, the SMA the basal ganglia, the insula, the thalamus and the dPFC. The left hand for the Inter-hemispheric condition results in privileged activations in a region of the dPFC (see 
Table 3). Relatively privileged representations of IT associated with the dominant hand are more strongly evident when considering analyses masked to white matter (Figure 5). 




It

(b) Inter-Hemispheric Transfer; LH $\neq$ RH



\section{Figure 5.}

Uni-manual effects in white matter are depicted for each of the experimental conditions: a) Within-hemispheric transfer and b) Inter-hemispheric transfer. Warm colors denote increased activation for the dominant right hand; cool colors denote increased activation for the nondominant left hand. Labels are intentionally withheld from the images to reduce clutter, but detailed statistical information and loci are presented in Tables $4 \mathrm{a}$ and $4 \mathrm{~b}$ respectively. As can be seen, the relative differences between uni-manual responses very strongly evident in the IT condition, where preferential activation is observed in longitudinal and transverse white matter clusters bilaterally (see Table 4). 


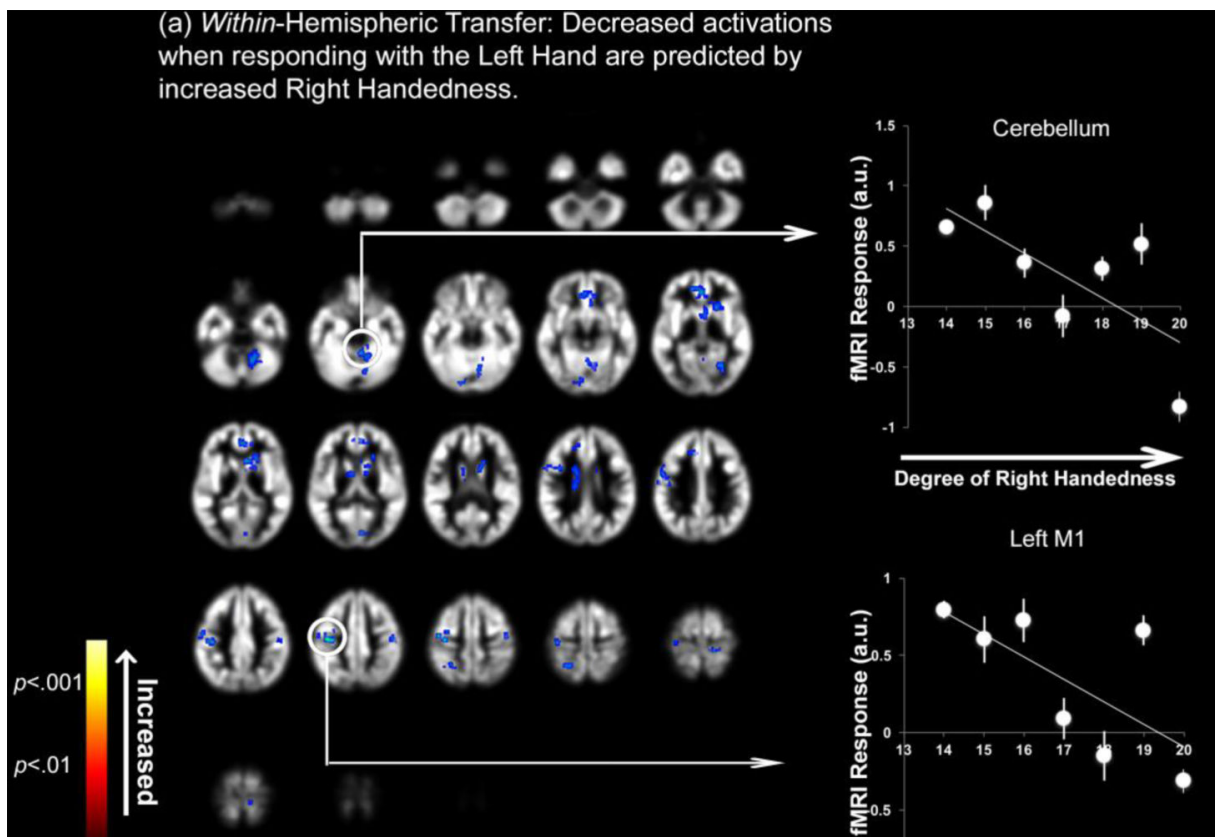

(b) Inter-Hemispheric Transfer: Decreased activations $p<.01$
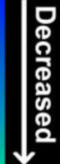

when responding with the Left Hand are predicted by increased Right Handedness.

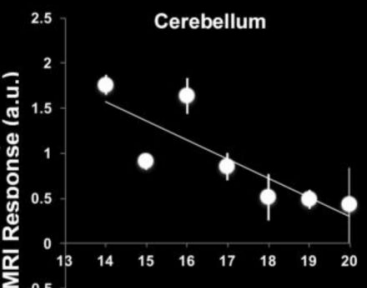

$-0.5$

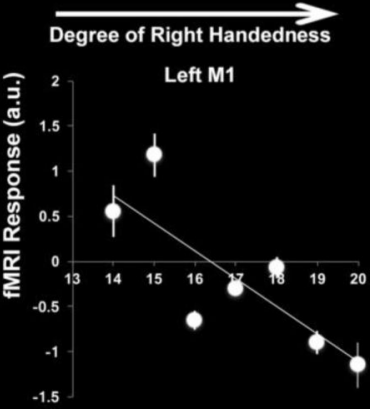

Figure 6.

Uni-manual responses with the left hand (Gray matter). The figure represents the results of a regression analysis examining the effects of the degree of right-handedness on activations during a) within- and b) inter-hemispheric transfer when responding with the non-dominant left hand. Only negative correlations were observed (cool colors), indicating that the degree of right-handedness was only associated with decreases in activation for responses with the left hand. Note that for the inter-hemispheric condition alone are effects strongly observed in contra-lateral (right) M1 as well. Each of the peaks in the ipsi- (M1) and contra-lateral (cerebellum) to the responding left hand are regions that are representative of responses with 
the right hand in these subjects (see Figure 4). These results suggest that representations of within and inter-hemispheric transfer when responding with the non-dominant left hand are sensitive to the degree of right-handedness. The degree of this sensitivity is greater for the inter- than the within-hemispheric condition (see Table 5 for statistical information). 

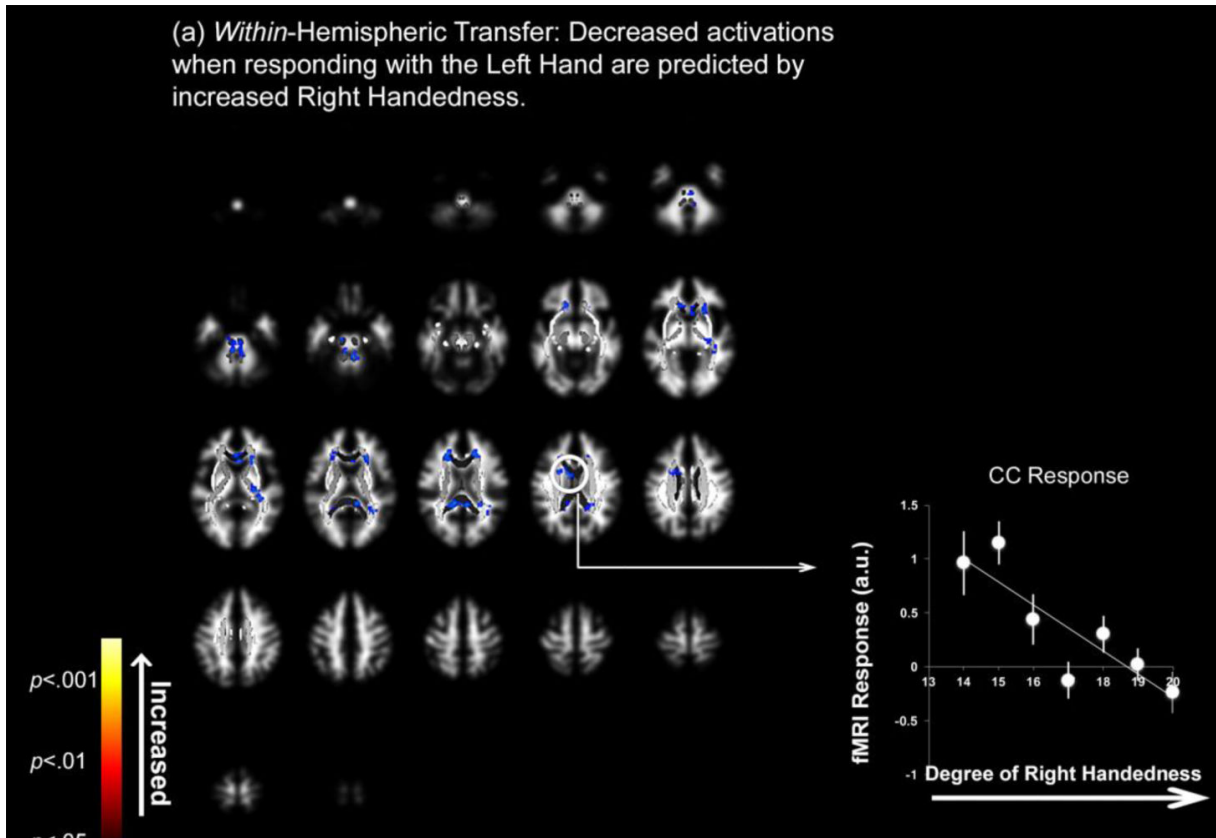

$p<.05$
$p<.01$
$p<.001$

(b) Inter-Hemispheric Transfer: Decreased activations when responding with the Left Hand are predicted by increased Right Handedness.
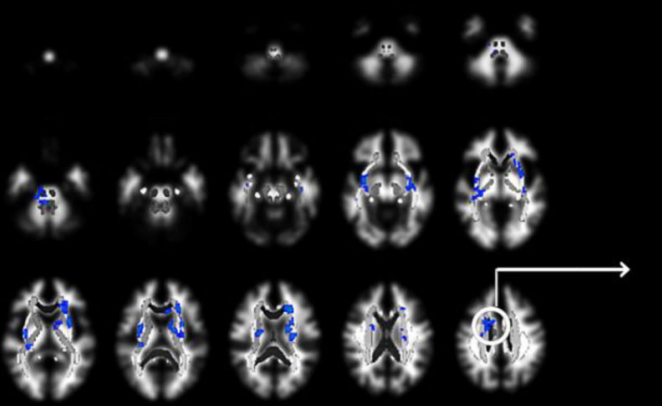
借非韭奜悲

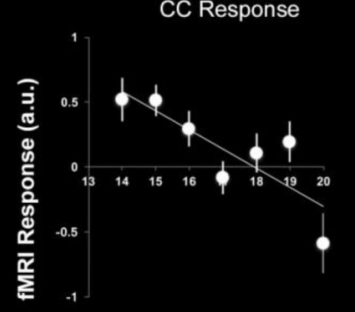

Degree of Right Handedness $\Rightarrow$

Figure 7.

Uni-manual responses with the left hand (White matter). The figure represents the results of a regression analysis examining the effects of the degree of right-handedness on activations during a) within- and b) inter-hemispheric transfer when responding with the non-dominant left hand. Only negative correlations were observed (cool colors) indicating that the degree of right-handedness was only associated with decreases in activation for responses with the left hand. The effects are respectively and selectively plotted for peaks in the corpus callosum. 


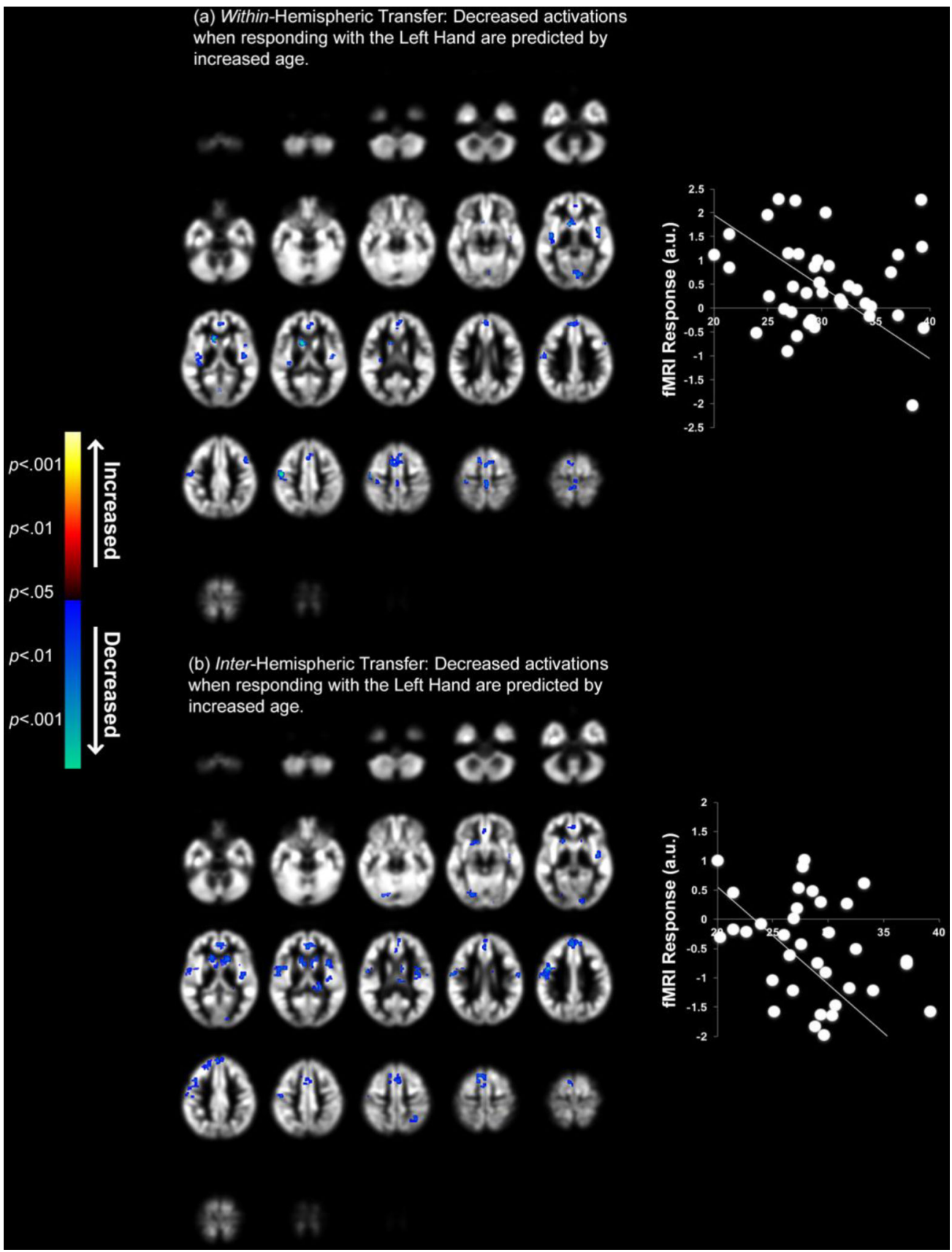

Figure 8.

Uni-manual responses with the left hand (Gray matter). The figure represents the results of a regression analysis examining the effects of age on activations during a) within- and b) interhemispheric transfer when responding with the non-dominant left hand. Only negative correlations were observed (cool colors), indicating that age was associated with decreases in activation for responses with the left hand. The graphs depict modeled fMRI responses under the overall peaks (see Table 7). 


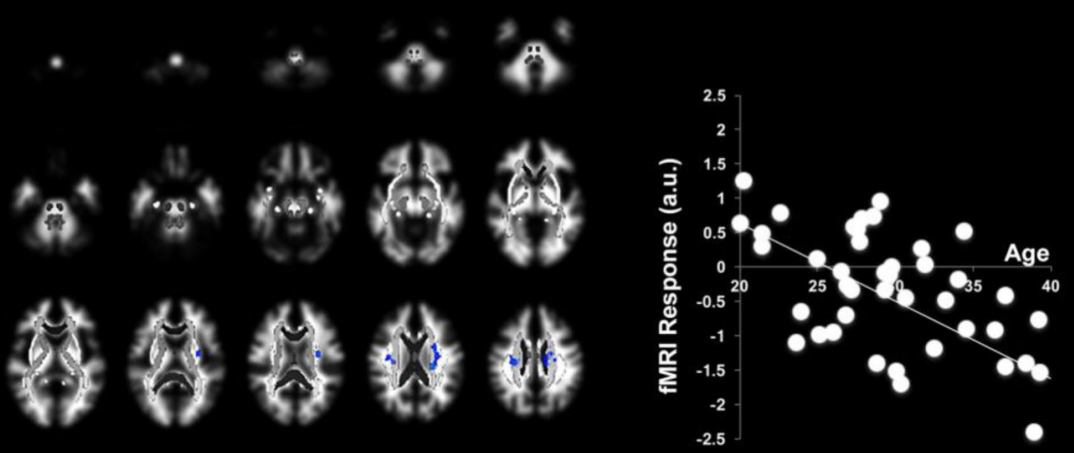

(b) Inter-Hemispheric Transfer: Decreased activations when responding with the Left Hand are predicted by increased aqe.
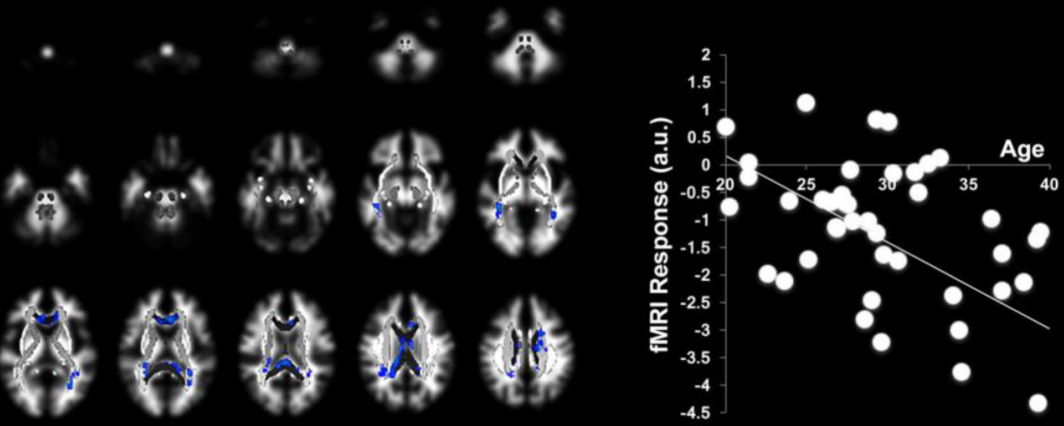

Figure 9.

Uni-manual responses with the left hand (white matter). The figure represents the results of a regression analysis examining the effects of age on activations during a) within- and b) inter-hemispheric transfer when responding with the non-dominant left hand. Only negative correlations were observed (cool colors), indicating that age was associated with decreases in activation for responses with the left hand. The graphs depict modeled fMRI responses under the overall peaks (see Table 8 ). 


\section{Table 1}

Demographics of the young adult, right-handed sample (total and for each gender sub-group), showing age, range and the mean handedness score (range: $14 \leq$ Score $\leq 20$ ).

\begin{tabular}{llll}
\hline & Age (sd) & Age Range (yrs.) & Handedness (sd) \\
Males $(\mathrm{n}=23)$ & $30.3(5.4)$ & $21.5-39.5$ & $17.4(2.0)$ \\
Females $(\mathrm{n}=26)$ & $30.5(6.2)$ & $20.1-40.8$ & $17.9(2.2)$ \\
Total $(\mathrm{n}=49)$ & $30.4(5.8)$ & $20.1-40.8$ & $17.7(2.1)$ \\
\hline
\end{tabular}




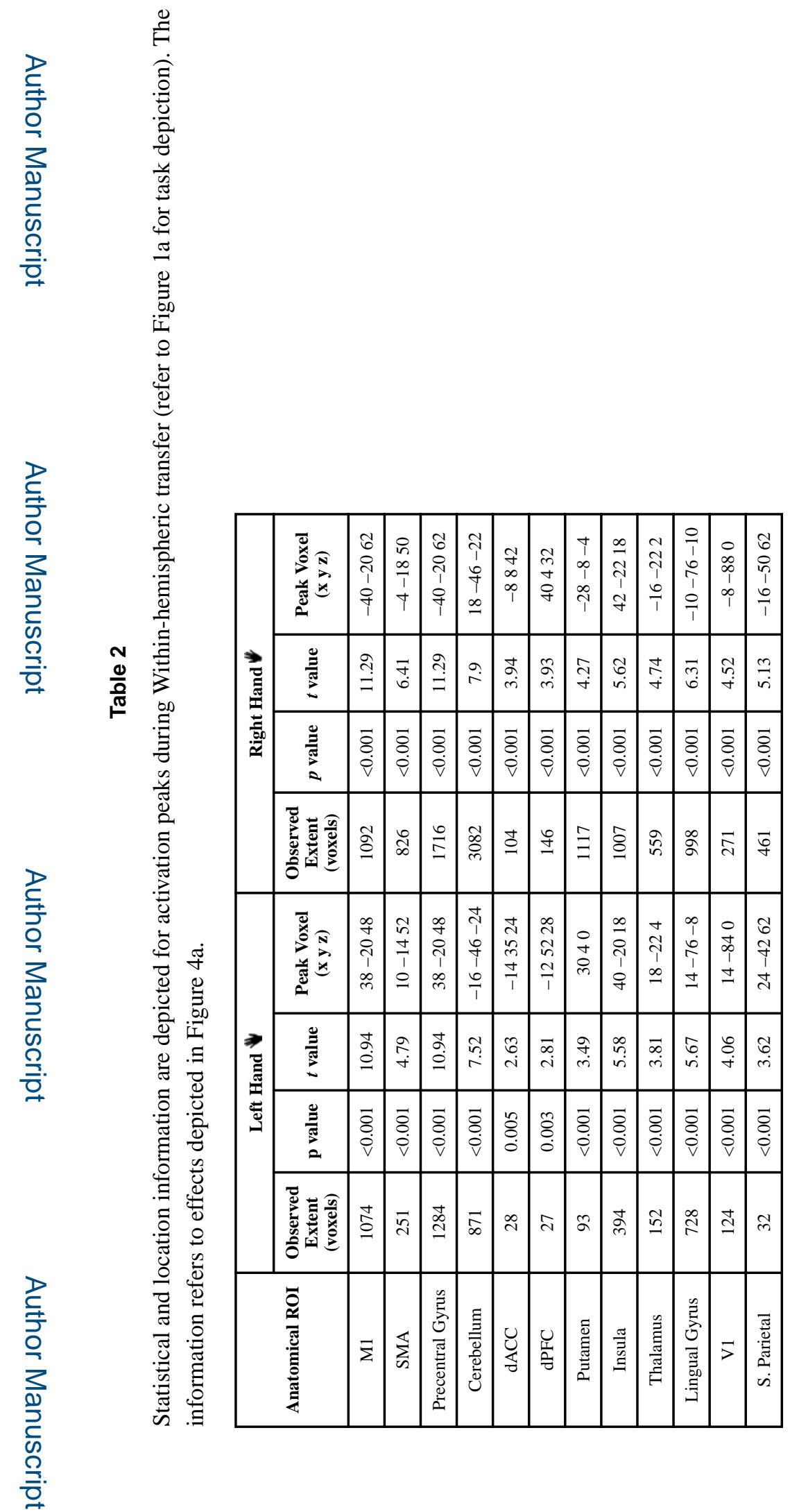

Brain Imaging Behav. Author manuscript; available in PMC 2019 August 01. 


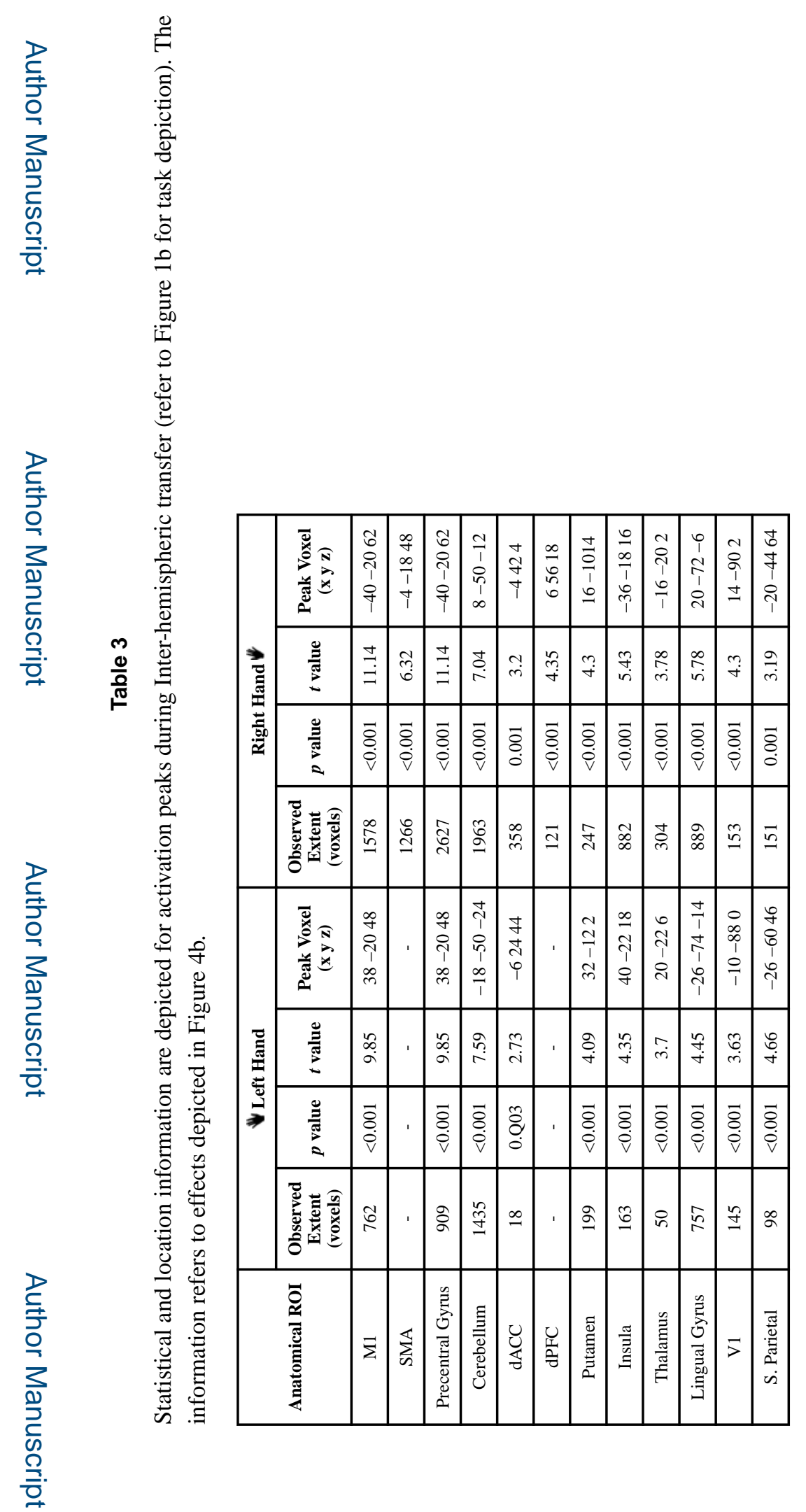

Brain Imaging Behav. Author manuscript; available in PMC 2019 August 01. 


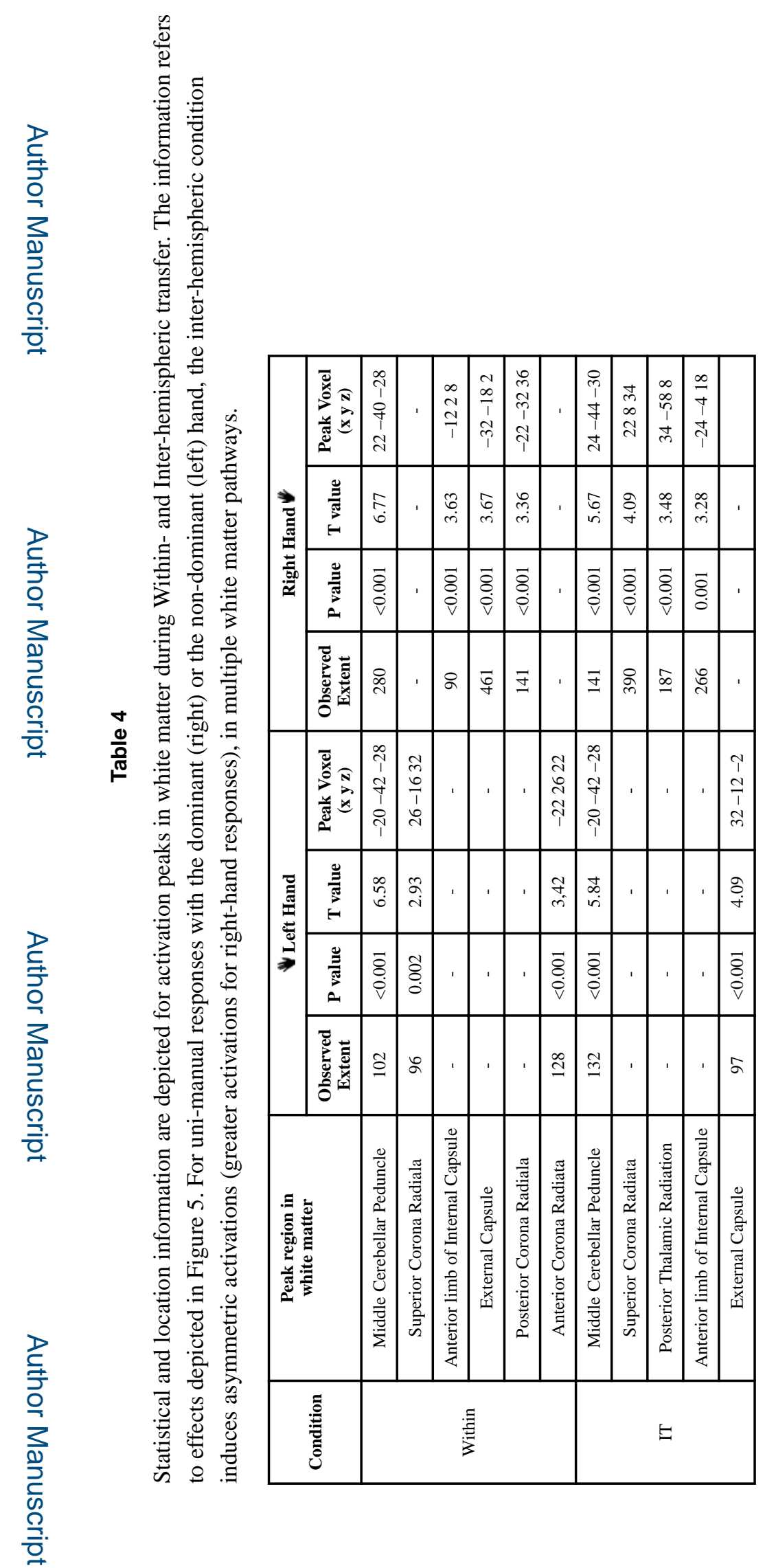

Brain Imaging Behav. Author manuscript; available in PMC 2019 August 01. 




Brain Imaging Behav. Author manuscript; available in PMC 2019 August 01. 


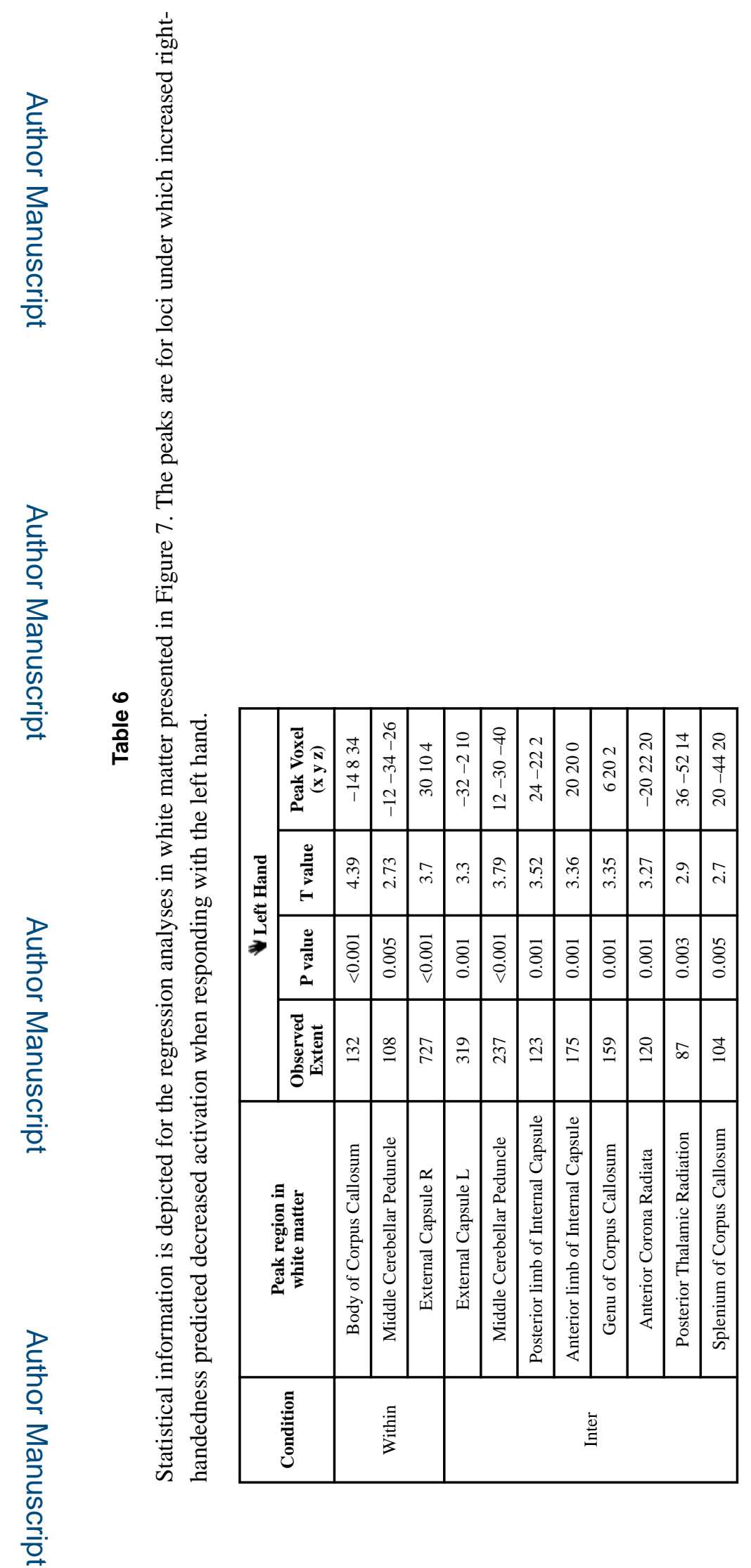

Brain Imaging Behav. Author manuscript; available in PMC 2019 August 01. 


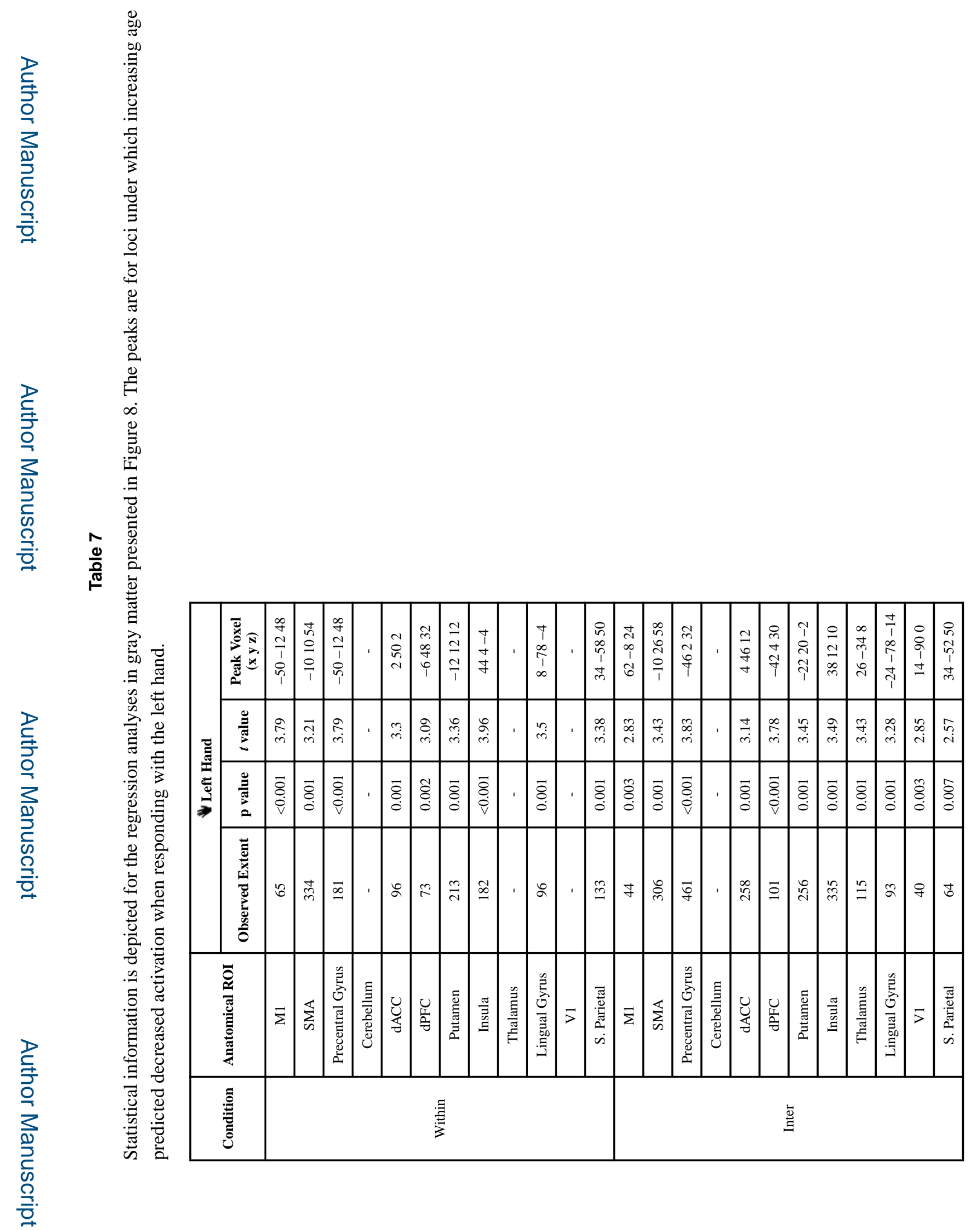

Brain Imaging Behav. Author manuscript; available in PMC 2019 August 01. 




Brain Imaging Behav. Author manuscript; available in PMC 2019 August 01. 